\title{
Chandra point-source counts in distant galaxy clusters ${ }^{\star}$
}

\author{
M. Branchesi ${ }^{1,2}$, I. M. Gioia ${ }^{2}$, C. Fanti ${ }^{2,3}$, R. Fanti ${ }^{2,3}$, and N. Cappelluti ${ }^{4}$ \\ 1 Dipartimento di Astronomia, Università di Bologna, via Ranzani 1, 40127 Bologna, Italy \\ e-mail: m.branchesi@ira.inaf.it \\ 2 Istituto di Radioastronomia, INAF, via Gobetti 101, 40129 Bologna, Italy \\ e-mail: gioia@ira.inaf.it \\ 3 Dipartimento di Fisica, Università di Bologna, via Irnerio 46, 40126 Bologna, Italy \\ e-mail: [rfanti;cfanti]@ira.inaf.it \\ 4 Max Planck Institute für Extraterrestrische Physik, \\ Postfach 1312, 85741, Garching, Germany \\ e-mail: cappelluti@mpe.mpg.de
}

Received 6 August 2006 / Accepted 24 September 2006

\begin{abstract}
Context. With the superb angular resolution of the Chandra Observatory, it is now possible to detect X-ray point sources, either embedded in galaxy clusters or along the cluster line of sight, which could not be resolved by previous instruments. This now allows studies of source counts in distant cluster fields.

Aims. We want to analyze the inner region of clusters of galaxies to check for the presence of any overdensity of X-ray point sources embedded in the gas diffuse emission. These point sources are possible AGN belonging to the clusters and could contaminate the cluster emission.

Methods. We used a sample of 18 distant $(0.25<z<1.01)$ galaxy clusters from the Chandra archive to construct the $\log N-\log S$, in both the soft and hard energy bands, for the X-ray point sources detected in the central cluster region to be compared with the counts of point sources detected in similarly deep fields without clusters.

Results. We find a $\sim 2 \sigma$ excess of cluster region sources at the bright end of the $\log N-\log S$. The radial distribution of the brightest $\mathrm{X}$-ray point sources confirms this excess and indicates that it is confined to the inner $0.5 \mathrm{Mpc}$ of the cluster region.

Conclusions. The results suggest the possible existence of X-ray sources belonging to the cluster (most probably AGN, given their $0.5-10 \mathrm{keV}$ luminosity ranging from $10^{43}$ to $10^{44} \mathrm{erg} \mathrm{s}^{-1}$ ): on average one every three clusters. Unlike previous studies, which have mainly investigated the point-source population in the vicinity of the galaxy clusters, the present study analyzes the content of point sources within the $1 \mathrm{Mpc}$ region covered by the cluster extent. Our work confirms the findings of other investigators who analyzed the central $1 \mathrm{Mpc}$ region of more massive clusters and/or groups in a similar redshift range. The X-ray source excess found here is much smaller than the excess of radio galaxies found recently in high- $z$ X-ray selected clusters, possibly due to the better sensitivity of the radio observations.
\end{abstract}

Key words. galaxies: clusters: general - galaxies: active - X-rays: general - X-rays: galaxies: clusters - galaxies: nuclei

\section{Introduction}

Clusters of galaxies harbor a wide diversity of galaxy populations, so they are ideal laboratories for studying galaxy formation and evolution. While there is clear evidence of the evolution in cluster galaxies (Butcher \& Oemler 1978, 1984), the evolution with redshift of cluster active galactic nuclei (AGN), as well as the possible prevalence of AGN in cluster environment with respect to the field, is still an unresolved issue. A related open debate is whether the cluster environment plays a role in the probability of galaxies to develop star-forming or AGN activity.

In the radio domain, searches for active galaxies in nearby galaxy clusters have been carried out for a long time (see among others Owen 1975; Fanti 1984; Ledlow \& Owen 1995a,b). The Radio Luminosity Function (RLF) of nearby cluster radio galaxies was found to be statistically indistinguishable from that of the field, both in shape and normalization (Ledlow \& Owen 1996). Recently, however, a radio survey of high redshift galaxy clusters

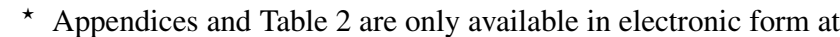
http://www . aanda.org
(Branchesi et al. 2006) has provided evidence of changes in the RLF of the distant cluster radio galaxies as compared to the local one. All these studies show that radio galaxies in clusters are strongly centrally concentrated and that their radial distribution essentially follows that of early type galaxies. As a result of the shape of the RLF and of the high sensitivity of radio telescopes, the typical radio luminosities found are not very high. Therefore the nuclear activity of these radio galaxies is not prominent at optical and/or X-ray wavelengths. On the other hand, studies from optical surveys seem to suggest that AGN are relatively rare in cluster environments. Cluster members that show evidence of AGN activity in their optical spectra are only $1 \%$ of all cluster galaxies, while AGN are more common (5\%) in the field population (Dressler et al. 1985). Furthermore, there is no evidence of any increase with redshift of the AGN fraction in clusters up to $z \sim 5$ (Dressler et al. 1999).

Since the pioneering work of Henry \& Briel (1991), who used ROSAT data to first reveal the presence of significantly more X-ray point sources around Abell $2256(z=0.06)$ than one would expect by chance, several studies have appeared in the literature that indicate $\mathrm{X}$-ray point-source excesses in the vicinity 
of low- and high- $z$ clusters with respect to the field. Many of these sources have been confirmed to be cluster members in several cases. At low redshift the excess seems to be largely due to the low-luminosity AGN $\left(\sim 10^{41} \mathrm{erg} \mathrm{s}^{-1}\right)$ associated with the cluster (Lazzati et al. 1998; Sun \& Murray 2002). At higher redshift, more luminous $\left(\sim 10^{42-43} \mathrm{erg} \mathrm{s}^{-1}\right)$ AGN are found in the fields of several clusters. It is evident from these studies that there is a population of obscured, or at least optically unremarkable, AGN in galaxy clusters. An AGN identification may not be obvious at optical wavelengths where the dusty AGN or those with weak emission lines may be not recognized as such (Martini et al. 2002). A more recent work by Martini et al. (2006), who completed a spectroscopic survey of X-ray point sources in eight low-redshift clusters $(z<0.3)$, finds that cluster galaxies host AGN more frequently than previously thought, a factor 5 higher than found by Dressler et al. (1985). Thus the optical spectroscopic surveys alone can underestimate the number of AGN in clusters. This is one of the reasons for the recently increased popularity of X-ray and radio wavebands for AGN detection at high redshift.

The capabilities of the current generation of X-ray observatories like Chandra (van Speybroeck et al. 1997) or XMM-Newton (Strüder et al. 2001; Turner et al. 2001) have triggered and extended to higher redshift these type of analyses (among others Cappi et al. 2001; Pentericci et al. 2002; Molnar et al. 2002; Cappelluti et al. 2005) thereby enabling studies with very fine spatial details. The superb angular resolution of the Chandra observatory, as well as the high sensitivity over the full X-ray band, now allow the detection of those X-ray point sources within the cluster region, which could not be resolved from the diffuse emission with previous instruments. The works mentioned above have shown an abundance of point sources in the direction of individual high- $z$ clusters as compared to the field. Different hypotheses for the apparent overdensities are examined by the different authors: 1) statistical variance of cosmic background sources; 2) gravitationally lensed background sources; 3) AGN/quasars and/or powerful starburst galaxies associated with the clusters. The last hypothesis is now considered the most plausible one.

Cappelluti et al. (2005) performed the first systematic search for X-ray point sources at the outskirts of 10 Chandra high- $z$ clusters $(0.24<z<1.2)$ and found a factor 2 overdensity $>2 \sigma$ significance level in $40 \%$ of clusters fields. They speculate that the most likely astrophysical interpretation of the overdensity is that the X-ray sources are AGN that trace the filaments connected to the clusters. This speculation is supported by the results of Cappelluti et al. (2007), who show that AGN and clusters are strongly correlated on scales between 2.5 and $10 \mathrm{Mpc}$ (smaller separations were not investigated). Further support for this interpretation comes from D'Elia et al. (2004), who studied the field surrounding the cluster 3C 295, which clearly exhibits a strong and asymmetric clustering of X-ray sources on scales of a few arcminutes.

Most of the above-mentioned studies analyzed the whole field around the clusters. In recent years it has become evident the importance of the location of AGN with respect to the cluster center, which could constrain different scenarios of AGN triggering. The study by Johnson et al. (2003) of MS 1054-03 at $z=0.83$, for instance, indicates that the X-ray AGN excess avoids the central regions of the cluster. Interestingly, AGN are not distributed randomly within the cluster but tend to populate the outer 1-2 Mpc, suggesting that AGN activity is triggered by recent infall at the cluster outskirt. In a recent work, Ruderman \& Ebeling (2005) study the spatial distribution of a sample of
$508 \mathrm{X}$-ray point sources detected in the soft $0.5-2.0 \mathrm{keV}$ band in Chandra observations of 51 clusters $(0.3<z<0.7)$ belonging to the MAssive Cluster Survey (MACS; Ebeling et al. 2001). The surface density of the X-ray point sources computed in the cluster rest frame exhibits an $8 \sigma$ excess within $3.5 \mathrm{Mpc}$ of the cluster centers. The authors resolve two distinct components of the excess, namely a central excess of AGN (at $\simeq 4 \sigma$ within $0.5 \mathrm{Mpc}$, as can be seen from their Fig. 2) and a broad secondary excess observed at about the virial radius, separated by a depletion region around $1.5 \mathrm{Mpc}$. They suggest that the central excess may be due to galaxy mergers and tidal interactions involving the central giant elliptical galaxies. The second excess could be caused by increased AGN activity at the cluster-field interface due to merger-induced accretion onto massive black holes.

In conclusion it seems that X-ray selected AGN are broadly distributed across the cluster. This could be a consequence of the different processes occurring close to the center of the cluster with respect to those occurring at the cluster-field interface. As mentioned earlier, radio galaxies are instead very centrally peaked. These two different distributions (X-ray AGN vs. radio galaxies) are not necessarily at variance since at the typical values of the radio luminosity (see for instance Falcke et al. 2004) the X-ray emission drops below the current X-ray detection levels.

In order to explore and investigate further whether and how the cluster environment plays a role in the statistical AGN properties of galaxies, the present paper focuses on the properties of point sources detected in the inner region of high- $z$ clusters using the X-ray energy band. Our X-ray analysis is limited to the central $\leq 1 \mathrm{Mpc}$ cluster region, so as to detect point sources embedded in the diffuse emission of the cluster gas. Since the number of sources expected in this area is not statistically significant for single cluster studies, we used a combined sample of eighteen clusters observed by Chandra. The redshift range is chosen to cover moderate to high redshift clusters $(0.25<z<1.01)$ in order to look for a possible redshift dependence of any excess found. All errors in this paper are at the $1 \sigma$ confidence level, unless otherwise noted. Throughout the paper, we use a $\Lambda$ CDM cosmology (Spergel et al. 2003) with $H_{0}=70 \mathrm{~km} \mathrm{~s}^{-1} \mathrm{Mpc}^{-1}$ and $\Omega_{\mathrm{m}}=1-\Omega_{\Lambda}=0.3$.

\section{The cluster sample}

The sample used in this study consists of 18 clusters of galaxies observed by the Chandra observatory with redshift in the range $z=0.25-1.01$. The choice of the redshift interval $(z>0.25$ and up to $z \sim 1$ ) was dictated by the requirement to select clusters that are reasonably matched in size by the field of view of the Chandra CCDs and, at the same time, at a cosmologically significant distance. We retrieved only those observations from the Chandra archive with exposure times greater than $30 \mathrm{ks}$ to be able to detect low surface-brightness clusters and the faint point sources projected against them. The choice of the exposure time was also dictated by the requirement of having enough count statistics to accurately measure the cluster temperatures that we will present in a future paper (Branchesi et al. in preparation) where the effect of the X-ray point sources on the cluster properties will be addressed. Table 1 lists the sample parameters and details of the Chandra observations. The columns contain the following information:

- Col. 1: cluster name;

- Col. 2: spectroscopic redshift tabulated in the literature; 
Table 1. Cluster sample parameters and details of Chandra observations.

\begin{tabular}{|c|c|c|c|c|c|c|c|c|c|c|}
\hline Cluster name & (2) & $\begin{array}{c}\text { RA } \\
\text { hh mm ss } \\
(3)\end{array}$ & $\begin{array}{c}\text { Dec } \\
\text { ' } \prime \\
(4)\end{array}$ & Obs. ID & ACIS & Mode & $\begin{array}{r}\text { Exp. } \\
\text { ks } \\
(8)\end{array}$ & $\begin{array}{c}N_{\mathrm{H}} \\
10^{20} \mathrm{~cm}^{-2} \\
(9)\end{array}$ & $\begin{array}{c}L_{0.5-2.0}^{\lim } \\
10^{42} \mathrm{cgs} \\
(10)\end{array}$ & $\begin{array}{l}L_{2.0-10.0}^{\lim } \\
10^{42} \mathrm{cgs} \\
\quad(11)\end{array}$ \\
\hline Abell 2125 & 0.246 & 154112 & +661601 & 2207 & I & VF & 79.7 & 2.77 & 0.13 & 0.56 \\
\hline ZW CL $1454.8+2233$ & 0.258 & 145715 & +222033 & 4192 & I & $\mathrm{VF}$ & 91.4 & 3.22 & 0.23 & 0.74 \\
\hline MS 1008.1-1224 & 0.302 & 101032 & -123923 & 926 & I & VF & 44.2 & 6.74 & 0.44 & 1.57 \\
\hline ZW CL $0024.0+1652$ & 0.394 & 002635 & +170939 & 929 & $S$ & VF & 36.7 & 4.19 & 0.34 & 2.22 \\
\hline MS 1621.5+2640 & 0.426 & 162336 & +263421 & 546 & I & $\mathrm{F}$ & 30.0 & 3.59 & 0.81 & 3.41 \\
\hline RXJ 1701.3+6414 & 0.453 & 170124 & +641410 & 547 & I & VF & 49.5 & 2.59 & 0.64 & 2.67 \\
\hline CL 1641+4001 & 0.464 & 164153 & +400146 & 3575 & I & VF & 44.0 & 1.02 & 0.67 & 2.62 \\
\hline V 1524.6+0957 & 0.516 & 152440 & +095748 & 1664 & I & VF & 49.9 & 2.92 & 0.89 & 3.29 \\
\hline MS 0451.6-0305 & 0.539 & 045412 & -030053 & 902 & $\mathrm{~S}$ & $\mathrm{~F}$ & 41.5 & 5.18 & 0.73 & 4.12 \\
\hline V $1121+2327$ & 0.562 & 112057 & +232627 & 1660 & I & VF & 66.9 & 1.30 & 0.73 & 3.00 \\
\hline MS 2053.7-0449 & 0.583 & 205621 & -043751 & 1667 & I & VF & 43.5 & 4.96 & 1.32 & 4.91 \\
\hline V $1221+4918$ & 0.700 & 122126 & +491830 & 1662 & I & VF & 79.4 & 1.44 & 1.18 & 4.62 \\
\hline MS 1137.5+6625 & 0.782 & 114022 & +660818 & 536 & I & VF & 117.5 & 1.18 & 0.81 & 4.04 \\
\hline RDCSJ 1317+2911 & 0.805 & 131721 & +2911 19 & 2228 & I & VF & 111.3 & 1.04 & 0.85 & 3.59 \\
\hline RDCSJ 1350+6007 & 0.805 & 135048 & +600654 & 2229 & I & VF & 58.3 & 1.76 & 1.77 & 7.26 \\
\hline RXJ 1716.4+6708 & 0.813 & 171649 & +670826 & 548 & I & $\mathrm{F}$ & 51.5 & 3.71 & 2.17 & 9.45 \\
\hline MS 1054.4-0321 & 0.830 & 105659 & -033737 & 512 & $\mathrm{~S}$ & $\mathrm{~F}$ & 67.5 & 3.67 & 1.07 & 6.61 \\
\hline WARPJ 1415.1+3612 & 1.013 & 141511 & +361200 & 4163 & I & VF & 89.2 & 1.10 & 1.93 & 7.54 \\
\hline
\end{tabular}

- Cols. 3-4: right ascension and declination (Equatorial J2000, HH MM SS.S, +DD MM SS.S) of the centroid of the Chandra photon distribution in the $0.5-5 \mathrm{keV}$ energy band assumed as the cluster center;

- Col. 5: identification number of the observation;

- Col. 6: detector where the aimpoint lies (I, for ACIS-I or S, for $A C I S-S)$;

- Col. 7: observation mode (F for FAINT or VF for VFAINT);

- Col. 8: exposure time in ks corresponding to the nominal exposure filtered to exclude time periods of high background;

- Col. 9: column density of Galactic hydrogen in units of $10^{20} \mathrm{~cm}^{-2}$, obtained from the Chandra X-ray Center (CXC) Proposal Planning Tool Colden (Galactic Neutral Hydrogen Density Calculator): NRAO-compilation by Dickey \& Lockman (1990);

- Col. 10: estimate of the luminosity limit for a cluster point source in erg s${ }^{-1}$ in the $0.5-2.0 \mathrm{keV}$ energy band. This limit is computed using the flux corresponding to $90 \%$ of the sky coverage of each cluster (see Appendix C for details);

- Col. 11: same as Column 10 but for the $2.0-10.0 \mathrm{keV}$ energy band.

With the exception of six clusters coming from the Einstein Medium Sensitivity Survey (EMSS; Gioia et al. 1990), all the clusters were originally discovered in ROSAT surveys, either the ROSAT All Sky Survey (Voges et al. 1999) or serendipitous surveys from pointed observations. Four clusters come from the 160 Square Degrees ROSAT Survey (Vikhlinin et al. 1998; Mullis et al. 2003), three from the ROSAT Deep Cluster Survey (RDCS; Rosati et al. 1998), one from the Wide Angle ROSAT Pointed Survey (WARPS; Perlman et al. 2002), and one, RXJ 1716+6708 (Gioia et al. 1999), is part of the NEP survey (Gioia et al. 2003; Henry et al. 2006). Three clusters, ZW CL 0024.0+1652, ZW CL 1454.8+2233, Abell 2125 are instead optically selected clusters.

\section{Source detection strategy}

Data reduction was performed using version 3.2.1 of the CIAO software (Chandra Interactive Analysis of Observations; see web page http://cxc.harvard.edu/ciao/index.html). The details are given in Appendix A.

For each ACIS CCD chip, two separate images were extracted from the event file for source detection at the raw resolution of $0.492 \mathrm{arcsec} \mathrm{pixel}^{-1}$. The two images are characterized by the following energies: a soft-energy image $(0.5-2.0 \mathrm{keV})$ and a hard-energy image $(2.0-7.0 \mathrm{keV})$. The cut-off below $0.5 \mathrm{keV}$ is necessary due to the steep drop off of the quantum efficiency and to the steep rise observed in the background rate due to charged particles. The cut-off above $7.0 \mathrm{keV}$ is necessary due to the decrease in the effective area of Chandra and to the increase in the instrumental background, which limits the detection efficiency of sky and source photons.

Sources were detected using the WAVDETECT algorithm (Freeman et al. 2002), included in the CIAO software package. The significance threshold used for source detection was set to the inverse of the total number of pixels, e.g. $\sim 10^{-6}$ for a $1024 \times$ 1024 pixels field. This is equivalent to stating that the expected number of false sources is one over the area of a single fullresolution ACIS chip. Wavelet scales were chosen in nine steps of $(\sqrt{2})^{i}$ pixel $(i=0, . ., 8)$ starting from $0.492^{\prime \prime}$, i.e. $\left(0.5^{\prime \prime}-8.0^{\prime \prime}\right)$ to cover a wide range of source sizes, accommodating extended sources and the variation in the PSF as a function of the offaxis angular distance, $\Theta$ (i.e. the distance of the source from the aimpoint).

The algorithm also uses an exposure map for each energy band to account for variations in the effective exposure across the $A C I S$ field of view. To consider the photon-energy dependence of the effective exposure time, exposure maps were created at a single energy resolution representative of the mean energy of the photons in each band: $1.0 \mathrm{keV}$ for the soft band and $4.0 \mathrm{keV}$ for the hard band.

\section{X-ray point-source sample}

Even if WAVDETECT is a detection algorithm, it supplies a list of source parameters that is very useful for photometric analysis. After running the algorithm on the different energy images, these parameters were used to build a sample of point sources. 
Following Manners et al. (2003) and Johnson et al. (2003), the source lists were built by accepting sources with a signalto-noise ratio $(S / N)$ greater than 3.0. This limit is a reasonably conservative one that guarantees the reliability of the sample sources. The source significance is defined as

$S / N=C /(1+\sqrt{0.75+B})$

where $C$ are the net source counts, and $B$ are the background counts within the "source cell", a region defined by WAVDETECT, which is assumed to contain all the source counts (Freeman et al. 2002). The denominator of Eq. (1) is an approximate expression for the error on the background counts from Gehrels (1986), who gives the upper confidence level equivalent to $1 \sigma$ Gaussian error for small number statistics. The definition of source significance is computationally convenient for defining a flux limit (see Appendix C) in each energy band. For a number of clusters we noticed that the detection algorithm tends to consider as point sources some slightly extended emission regions close to the cluster center. In most cases, these sources are simply cluster clumps of the thermal gas rather than central X-ray point sources. A visual inspection of all the detected sources enabled us to tentatively eliminate these dubious point source identifications from the sample.

A total of 119 X-ray sources were detected in the searched cluster area. Of these, 41 sources were detected only in the soft band, 24 only in the hard band, and 54 are common to both bands. The search radius $R_{\text {ext }}$ (listed in Table 2 for each cluster) is the radius that includes the cluster region where diffuse emission is still detectable. It is namely the radius at which the cluster surface-brightness profile merges into the background, and beyond which no further significant cluster emission is detectable. The total area covered by the clusters is $\sim 0.083 \mathrm{deg}^{2}$. The survey is complete down to a flux limit of $2.7 \times 10^{-15} \mathrm{erg} \mathrm{cm}^{-2} \mathrm{~s}^{-1}$ in the soft energy band and to $0.8 \times 10^{-14} \mathrm{erg} \mathrm{cm}^{-2} \mathrm{~s}^{-1}$ in the hard energy band, corresponding to $100 \%$ of the respective sky coverages (see Appendix C). However there are a number of individual clusters surveyed to a fainter point source flux.

The source list is given in Table 2. A detailed description of how the X-ray source parameters are computed is given in Appendix B. The columns of Table 2 contain the following information:

- Col. 1: cluster name;

- Col. 2: search radius, $R_{\text {ext }}$, in arcsec;

- Col. 3: source identification number;

- Cols. 4-5: WAVDETECT source position; Right Ascension and Declination (Equatorial J2000, HH MM SS.S, + DD MM SS.S);

- Cols. 6-7: net counts in the soft $(0.5-2.0 \mathrm{keV})$ and hard (2.0-7.0 keV) energy bands. Asterisks indicate sources not used in Sect. 5 for the computation of the source counts since their flux is smaller than the flux corresponding to $20 \%$ of the sky coverage (see Appendix C);

- Col. 8: observed X-ray flux in units of $10^{-15} \mathrm{erg} \mathrm{cm}^{-2} \mathrm{~s}^{-1}$ in the soft $(0.5-2.0 \mathrm{keV})$ band. The flux and the associated error have been calculated as described in Appendix B;

- Col. 9: correction factor to be applied to the observed soft $\mathrm{X}$-ray flux to obtain the Galactic absorption corrected X-ray flux;

- Col. 10: same as Col. 8 but for the hard (2.0-10.0 keV) band.

\section{X-ray point-source number counts}

We used the source counts, or $\log N-\log S$ relationship, as a statistical tool for investigating whether there is any point source count excess in the regions of diffuse cluster emission with respect to the fields void of visible clusters. Although integral source counts are usually given in the literature, we present here both the differential and integral distributions. The differential number counts are statistically more correct since source-count errors are independent, differently from the integral number counts. To compute the $\log N-\log S$ relationship, we followed the method described in Gioia et al. (1990). The source contributions are computed by properly weighting each source with its visibility area, i.e. the area of sky where the source intensity equals or exceeds the sensitivity limit (see Appendix $\mathrm{C}$ for details). The integral $\log (N>S)$ diagram is then built by summing, in decreasing flux order, the contribution from each source. Since each cluster sky coverage rapidly decreases near the limiting flux for both the soft and hard bands, in order to prevent incompleteness effects, we considered only those sources with a flux larger than the flux corresponding to $20 \%$ of the total sky area covered by each cluster. It is worth noting that, throughout the following sections, the soft X-ray fluxes are unabsorbed fluxes, i.e. corrected for the Galactic hydrogen column density absorption along the line of sight at each field position.

\subsection{X-ray source counts in the cluster regions}

Following Crawford et al. (1970) and Murdoch et al. (1973), a Maximum Likelihood Method (MLM) that operates on the differential counts, was adopted to determine the slope of the number-flux distribution of the X-ray sources. The first assumption is that the differential $\log N-\log S$ distribution may be described by a single power-law model of the form:

$\frac{\mathrm{d} N}{\mathrm{~d} S}=b\left(\frac{S}{S_{0}}\right)^{-\alpha 1}$.

The MLM uses the "unbinned" data that is very useful when one deals with a low number of sources.

The MLM applied to the sources found in the regions covered by our clusters (from now on cluster region sources) provides the following results:

$\frac{\mathrm{d} N}{\mathrm{~d} S}=354 \pm 37\left(\frac{S}{2 \times 10^{-15} \mathrm{erg} \mathrm{cm}^{-2} \mathrm{~s}^{-1}}\right)^{-1.78+0.08}$ sources $\operatorname{deg}^{-2}(3)$

for the $0.5-2.0 \mathrm{keV}$ band and

$\frac{\mathrm{d} N}{\mathrm{~d} S}=255 \pm 29\left(\frac{S}{1 \times 10^{-14} \mathrm{erg} \mathrm{cm}^{-2} \mathrm{~s}^{-1}}\right)^{-2.0 \pm 0.1}$ sources deg ${ }^{-2}$

for the $2.0-10.0 \mathrm{keV}$ band.

The values for $b$ were calculated at the flux levels of $S_{0}=$ $2 \times 10^{-15} \mathrm{erg} \mathrm{cm} \mathrm{cm}^{-2} \mathrm{~s}^{-1}$ in the soft band and $S_{0}=1 \times$ $10^{-14} \mathrm{erg} \mathrm{cm}^{-2} \mathrm{~s}^{-1}$ in the hard band. For the exposures considered here, these values correspond to the centers of the sampled flux intervals.

The differential logarithmic source counts, in bins of $\Delta \log S=0.2$, are indicated in Fig. 1 by filled triangles. The soft (hard) $\log N-\log S$ for the X-ray point sources detected in the areas covered by the clusters is given in the top (bottom) panel. The counts and the errors are given by:

$\mathrm{d} N=\sum_{i=1}^{n} \frac{1}{\Omega_{i}} \operatorname{deg}^{-2}$ and $\sigma_{\mathrm{d} N}=\sqrt{\sum_{i=1}^{n}\left(1 / \Omega_{i}^{2}\right) \mathrm{deg}^{-2}}$ 

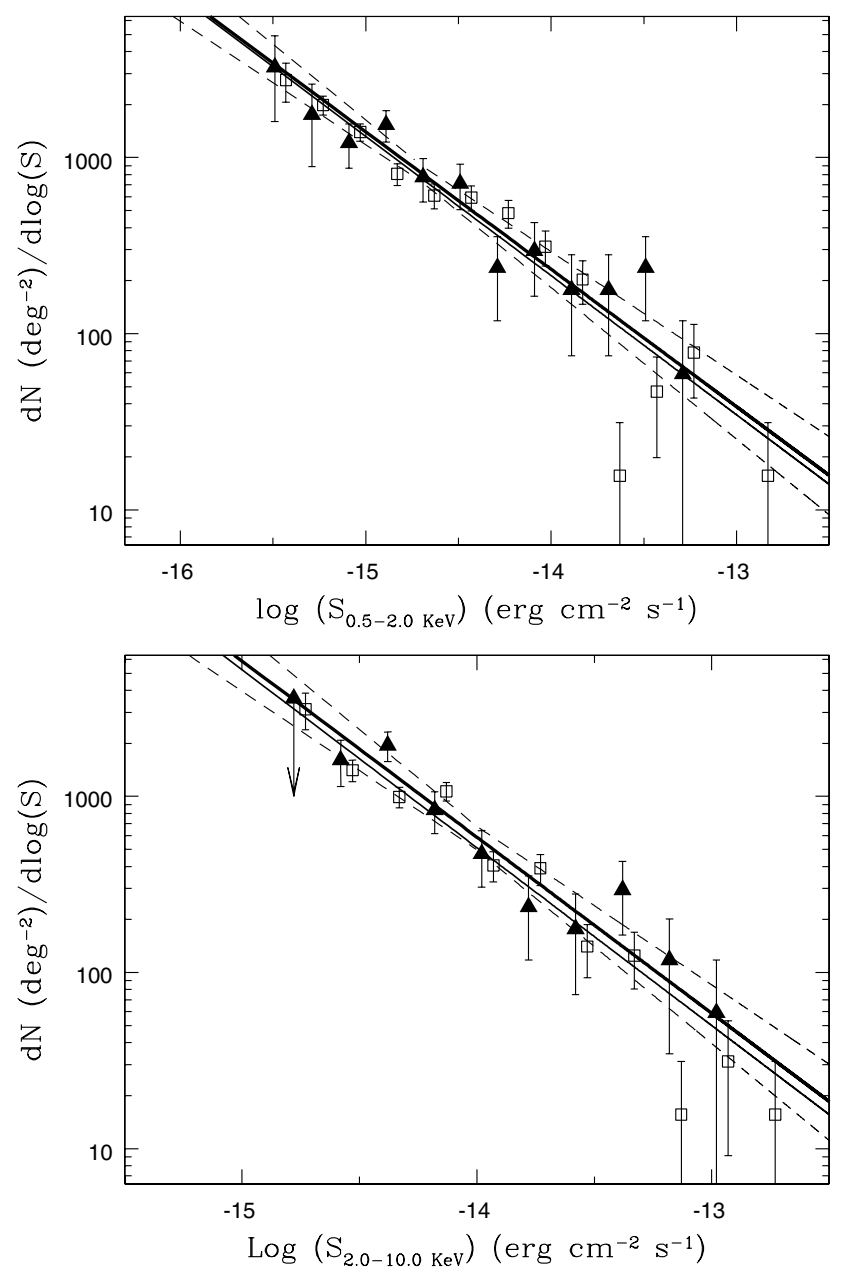

Fig. 1. Differential $\log N-\log S$ s. The differential $\log N-\log S$ of the $\mathrm{X}$-ray point sources detected in areas covered by the clusters are indicated by solid triangles (soft band in the top panel and hard band in the bottom panel). Open squares indicate the differential $\log N-\log S$ of the X-ray point sources detected in the field obtained by combining the four deep $(\tau \sim 100 \mathrm{ks})$ fields without clusters (Bootes, CDFS, HDFN, and Groth Strip). The uncertainty on each point corresponds to a $1 \sigma$ confidence level. No cluster region sources are observed in the hard faintest flux bin. The arrow indicates an upper limit corresponding to three sources. According to Poisson statistics, we have a 5\% probability of observing zero sources when three are expected. The thick (thin) solid lines represent the best-fit power law of the $\log N-\log S$ for the cluster region (field) sources obtained with the MLM, while the dashed lines correspond to the $1 \sigma$ uncertainties on the fit for the cluster region sources. The uncertainties on the field source fit are smaller and have been omitted for clarity.

where $n$ is the number of sources within each bin and $\Omega_{i}$ the visibility area of the $i$ th source. The thick solid lines represent the best fit obtained with the MLM described above. The dashed lines indicate the $1 \sigma$ uncertainties on the MLM fit parameters.

To compare our results with those in the literature, we also calculated the cumulative source number counts $N(\geq S)$ and the corresponding errors as follows:

$N(>S)=\sum_{i=1}^{N_{\text {tot }}} \frac{1}{\Omega_{i}} \operatorname{deg}^{-2}$ and $\sigma_{N(>S)}=\sqrt{\sum_{i=1}^{N_{\text {tot }}}\left(1 / \Omega_{i}^{2}\right)} \operatorname{deg}^{-2}(6)$

where $N_{\text {tot }}$ is the total number of detected sources with a flux $\geq S$ and $\Omega_{i}$ the sky coverage corresponding to the flux of the
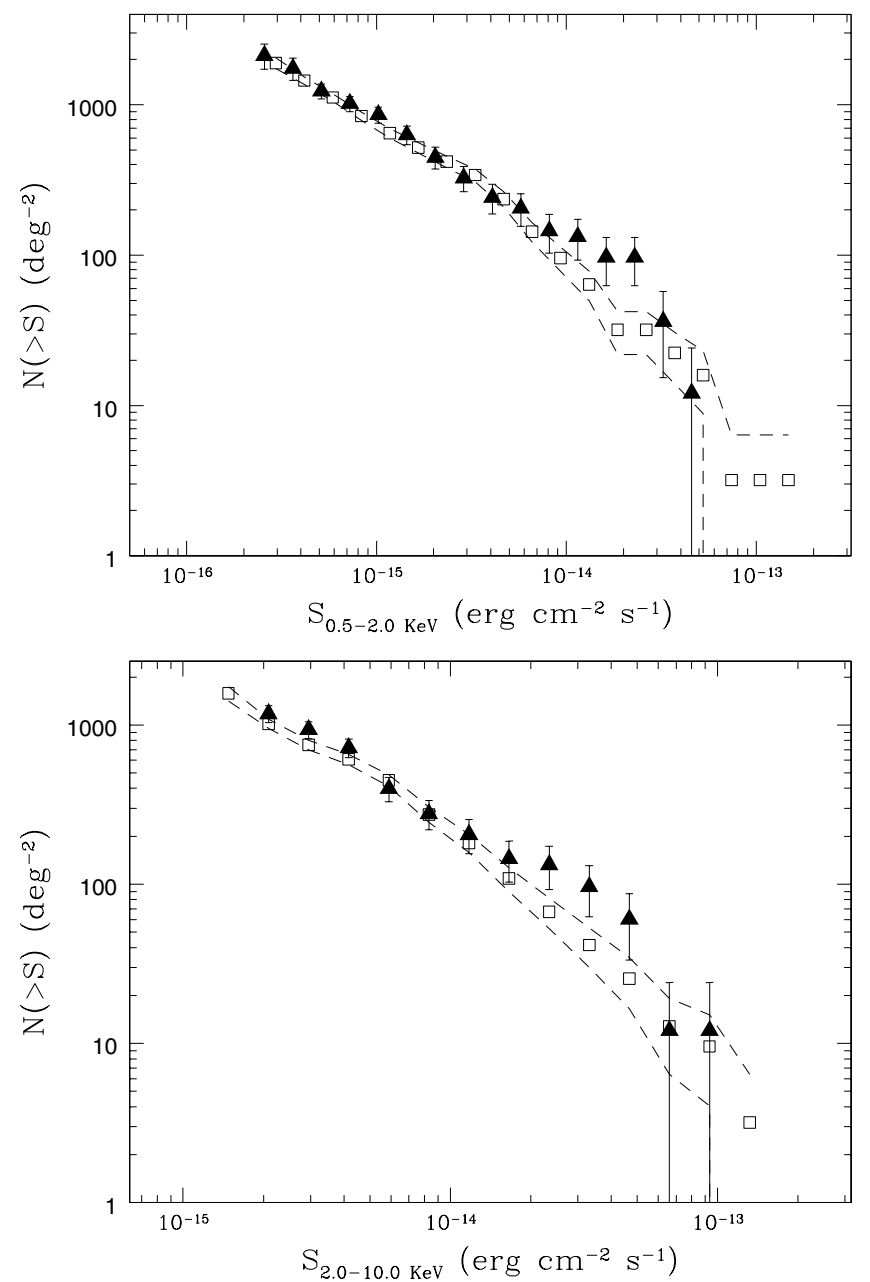

Fig. 2. Integral $\log N-\log S \mathrm{~s}$. The integral $\log N-\log S$ for the sources detected in the regions occupied by clusters are indicated by filled triangles in both the soft (top panel) and hard (bottom panel) energy band. Open squares indicate the integral $\log N-\log S$ for the sources detected in the unified reference field obtained by combining the four $\sim 100 \mathrm{ks}$ long fields without visible clusters (Bootes, CDFS, HDFN, and Groth Strip). The cumulative source counts are plotted using a step $\Delta \log S=0.15$. Uncertainties on each $\log N-\log S$ point correspond to a $1 \sigma$ confidence level obtained as described in the text. Dashed lines represent $1 \sigma$ confidence level for the field sources counts.

$i$ th source. The assumption made of a single power law converts Eq. (2) into

$N(>S)=k\left(\frac{S}{S_{0}}\right)^{-\alpha}$

for the integral distribution, where $\alpha=\alpha 1-1$. The integral normalization $k$ is calculated by integrating Eq. (2) between the flux limit and infinity:

$k=\frac{b}{\alpha 1-1}$.

The errors on $k$ were calculated using the propagation of error for $\alpha 1$ and $b$. The results for the integral source counts in the cluster fields are listed in Table 3.

Figure 2 illustrates the cumulative source counts, indicated by solid triangles, which were plotted using a step of $\Delta \log S=$ 0.15 . The soft integral $\log N-\log S$ is shown in the top panel and the hard integral $\log N-\log S$ in the bottom panel. 
Table 3. Integral source counts in clusters and in reference fields.

\begin{tabular}{|c|c|c|c|c|c|c|c|c|c|}
\hline Name & Obs. Id & $\begin{array}{c}\tau \\
\text { ks } \\
\text { (3) }\end{array}$ & $\begin{array}{c}N_{\mathrm{H}} \\
\mathrm{cm}^{-2} \\
(4)\end{array}$ & $N_{0.5-2.0}$ & $N_{2.0-7.0}$ & $\alpha_{0.5-2.0}$ & $\alpha_{2.0-10.0}$ & $\begin{array}{c}k_{0.5-2.0} \\
\mathrm{deg}^{-2} \\
(9)\end{array}$ & $\begin{array}{c}k_{2.0-10.0} \\
\mathrm{deg}^{-2} \\
(10)\end{array}$ \\
\hline Clusters & & & & 92 & 77 & $0.78 \pm 0.08$ & $1.00 \pm 0.10$ & $454 \pm 66$ & $255 \pm 39$ \\
\hline Bootes & 313 & 10 & 1.15 & 90 & 84 & & & $410 \pm$ & $217 \pm$ \\
\hline & 3389 & 109 & 1.50 & 9 & 6 & & & & \\
\hline & & 128 & 0.80 & 109 & 8 & & & & \\
\hline Groth & 4357 & 85 & 1.30 & 83 & 52 & $0.80 \pm 0.08$ & $0.94 \pm 0.12$ & $432 \pm 63$ & $217 \pm 41$ \\
\hline Unified & & & & 378 & 280 & $0.79 \pm 0.04$ & $1.01 \pm 0.04$ & $416 \pm 30$ & $219 \pm 16$ \\
\hline
\end{tabular}

- Col. 1: reference field name;

- Col. 2: identification number of the observation;

- Col. 3: total net exposure time after "flare" cleaning;

- Col. 4: hydrogen Galactic column density value, $N_{\mathrm{H}}$, in units of $10^{20} \mathrm{~cm}^{-2}$.

- Cols. 5-6: number of sources detected in the soft and hard bands with flux larger than the flux corresponding to $20 \%$ of the sky coverage;

- Cols. 7-8: the integral $\log N-\log S$ power law slope and $1 \sigma$ confidence level error for the soft and hard bands;

- Cols. 9-10: the integral $\log N-\log S$ normalization and $1 \sigma$ confidence level error calculated in the soft band at a flux of $2 \times 10^{-15} \mathrm{erg} \mathrm{cm}^{-2} \mathrm{~s}^{-1}$ and in the hard band at a flux of $1 \times 10^{-14} \mathrm{erg} \mathrm{cm}^{-2} \mathrm{~s}^{-1}$. Note that the soft band normalization takes into account the different Galactic hydrogen column density.

Errors correspond to $1 \sigma$ confidence level and are obtained using Eq. (6). Note that the errors on the integral $\log N-\log S$ are not independent.

\subsection{X-ray source counts in the reference fields}

The slope and/or normalization of the source counts at a given flux limit can provide information on the point-source population in the cluster regions compared to the sources in fields without visible clusters (from now on field sources). To check for the presence of such an effect, the results obtained in the area of the 18 clusters were compared with those obtained in four reference fields void of clusters with exposure times similar to the exposures of the cluster fields.

The reference fields used were the Chandra Deep Field South (CDFS), the Hubble Deep Field North (HDFN), the Groth/Westphal strip area, and the Bootes field (see Table 3). In order to sample similar flux levels, the analysis was limited to observations with exposure on the order of $100 \mathrm{ks}$. The analysis was performed on the four ACIS-I CCD $\left(16.8^{\prime} \times 16.8^{\prime}\right)$. For the validity of our analysis and to avoid systematic errors, we ran exactly the same procedure on the reference fields as was used to derive the $\log N-\log S$ of the cluster region sources. The same source-detection routine, flux limit and flux estimate method have been adopted (see Sects. 3 and 4 and Appendix for details). The sky coverage of each reference field was constructed following the method described in Appendix C. The only difference is the binning of the background and exposure maps, which were chosen to be $64 \times 64$ pixels to reduce the computer time. The value of the flux limit of each bin is a representative mean of all the original pixels since the absence of a galaxy cluster in the field results in a much more uniform background. The sky area of each field measures $\sim 0.078 \mathrm{deg}^{2}$. Following the same approach as the cluster region sources we considered only field sources with a flux greater than the flux corresponding to $20 \%$ of the sky coverage of each field.

The best-fit results on the cumulative $\log N-\log S$ in each reference field are summarized in Table 3 . Note that the literature reports source counts that are usually estimated using the observed fluxes, while we corrected for the Galactic hydrogen column density to produce unabsorbed fluxes. The counts agree with recent studies of these fields (Giacconi et al. 2001; Tozzi et al. 2001; Rosati et al. 2002; Brandt et al. 2001; Wang et al. 2004). The slope values match the published ones within the errors. The normalizations are also consistent within the errors, considering that we used different, more recent calibrations and a different spectral slope $(\Gamma=1.7)$ to derive the conversion factors (see Appendix B). Most of the authors quoted above use a flatter slope with $\Gamma=1.4$. The average difference between the conversion factors obtained considering an absorbed power law spectrum with $\Gamma=1.7$ and $\Gamma=1.4$ are

$$
\begin{aligned}
& K_{\text {soft }}(1.4) \sim K_{\text {soft }}(1.7)-3 \% K_{\text {soft }}(1.7) \\
& K_{\text {hard }}(1.4) \sim K_{\text {hard }}(1.7)+12 \% K_{\text {hard }}(1.7)
\end{aligned}
$$

Finally the four reference fields were combined in a unique big field of $\sim 0.31 \mathrm{deg}^{2}$. A corresponding sky coverage was also constructed. The 378 plus 280 sources, detected in the soft and hard band respectively, have been used to derive both the differential and integral $\log N-\log S$ for the unified reference field. The data were best-fitted using the MLM. The best-fit integral source count parameters are listed in Table 3.

Hereafter the unified reference field is used for comparison with the cluster fields, but the four single reference fields give a measure of the parameter dispersion. The differential source counts in bins of $\Delta \log S=0.2$ for the unified reference field are indicated by open squares in Fig. 1. The errors correspond to $1 \sigma$ confidence level obtained as in Eq. (5). The thin solid lines represent the power law corresponding to the best-fit parameters obtained with the MLM.

The cumulative source counts of the unified reference field are shown in Fig. 2 (open squares). The dashed lines represent $1 \sigma$ uncertainties on the source counts.

\section{Are X-ray point sources associated with the clusters?}

\subsection{Cluster counts vs. field counts}

Figure 3 illustrates the best-fit normalization and slope of the integral $\log N-\log S \mathrm{~s}$, for both the cluster and the reference field sources. The parameters of the fits, given in Table 3, were 

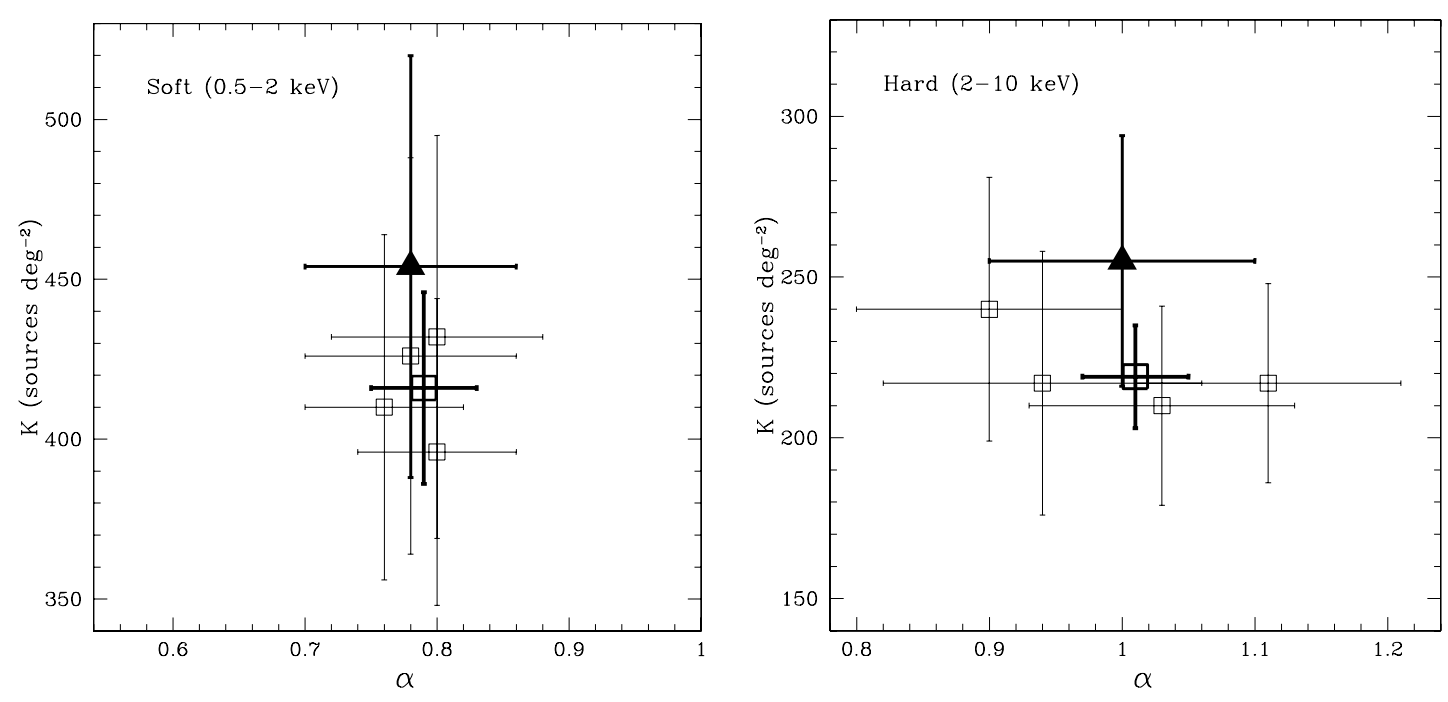

Fig. 3. Integral $\log N-\log S$ normalization vs. slope for both cluster region and field sources obtained using the MLM. Solid triangles indicate the best-fit results for the cluster region sources (soft band to the left and hard band to the right), while the thick open squares indicate the unified reference field results. The thin open squares indicate the best-fit results for each of the four reference fields. Uncertainties correspond to a $1 \sigma$ confidence level on the fit parameters.

obtained by fitting one single power law over the entire flux range. All values are consistent within $1 \sigma$ confidence level in both bands, showing no significant difference between the counts in the cluster areas and in the control fields. However, an inspection of the top panel of Fig. 2 shows that the soft integral cluster counts exhibit a small excess at the bright end around $\sim 2 \times 10^{-14} \mathrm{erg} \mathrm{cm}^{-2} \mathrm{~s}^{-1}$. A similar excess is present in the hard integral number counts (Fig. 2, bottom) around $\sim 3.0 \times$ $10^{-14} \mathrm{erg} \mathrm{cm}^{-2} \mathrm{~s}^{-1}$. The significance of these apparent excesses was evaluated by computing the number of field point sources expected in the cluster regions. Since the field source counts in both bands show a steeper slope at the bright end compared to the faint end, as also found by several authors (Hasinger et al. 1998; Mushotzky et al. 2000; Tozzi et al. 2001; Rosati et al. 2002), instead of using the single power-law best fit, we considered it more appropriate computing the expected number of field sources by directly normalizing the number of the observed point sources in the control fields to the area covered by the clusters. We would expect $5 \pm 1$ field sources above a flux of $1.25 \times 10^{-14} \mathrm{erg} \mathrm{cm}^{-2} \mathrm{~s}^{-1}$ for the soft band and the same number above $2.5 \times 10^{-14} \mathrm{erg} \mathrm{cm}^{-2} \mathrm{~s}^{-1}$ for the hard band. We instead find 11 sources in the cluster region in each band. The significance of the difference is at the $1.7 \sigma$ level in both cases. Note that 9 sources out of the 11 are common to both bands. If this small excess is real, we are facing a cluster population of $\mathrm{X}$-ray sources with X-ray luminosities ranging from $\approx 3 \times 10^{42}$ to $10^{44} \mathrm{erg} \mathrm{s}^{-1}$ in the soft band and from $\approx 10^{43}$ to $10^{44} \mathrm{erg} \mathrm{s}^{-1}$ in the hard band.

Next we examined whether there is any dependence of the number counts on cluster redshift. For this purpose the point source sample was divided into two subsamples: the "high-z" subsample corresponding to the sources detected in the regions covered by clusters with redshift $z>0.7$ and the "low- $z$ " subsample corresponding to the sources detected in the regions covered by clusters with redshift $z<0.7$. We performed an analysis on these two subsamples similar to the one performed on the whole sample. No significant difference between the two subsamples is evident in the soft band. In the hard band there is instead a slight indication of a possible excess at bright fluxes in the "high- $z$ " subsample with respect to the "low- $z$ " one. The statistics are too poor to draw any conclusion.
Finally it is interesting to compare our estimate of the "cluster X-ray population" with the results by Martini et al. (2006) for a sample of nearby clusters. Under the assumption that our clusters are similar to the Martini clusters, we used their spectroscopically confirmed cluster X-ray sources to derive a "Cluster X-ray Luminosity Function" (Cl-XLF) and predict how many cluster $\mathrm{X}$-ray sources we should expect in our sample. In their five clusters with $z>0.15$, Martini et al. (2006) list seven objects within $1 \mathrm{Mpc}$ from the cluster center with a soft $L_{x}>10^{42} \mathrm{erg} \mathrm{s}^{-1}$, while no object is present in the three closer clusters with redshifts of $0.07,0.06$, and 0.059 . If we build the Cl-XLF from the five $z>0.15$ clusters by properly accounting for our luminosity limits, we expect five cluster X-ray sources, four of which are within $0.5 \mathrm{Mpc}$ and one between 0.5 and $1 \mathrm{Mpc}$ from the cluster center ${ }^{1}$. These expected numbers agree with the numbers of sources in clusters previously estimated from our own data. Had we used the data from all the Martini eight clusters, the Cl-XLF would have had a lower amplitude, and the predicted number of cluster X-ray sources in our sample would have dropped to $\leq 4$. A similar analysis cannot be performed in the hard band due to the presence of only two X-ray sources in Martini et al. (2006) work.

\subsection{Radial distribution of the point sources}

To further investigate the reality of the excess at high flux, we examined the radial distribution of the brightest sources in both bands as a function of the projected linear distance from the cluster center. Given the small number of sources, only two $0.5 \mathrm{Mpc}$ wide annuli were used. From the control field source counts, normalizing to the areas actually examined, we computed the expected number of field sources in the two cluster annuli. The results are listed in Table 4 (Cols. 2 and 5). In each band 5 field sources are expected in the area covered by the clusters (see Sect. 6.1). Two field sources are expected in the inner annulus against 7 observed, and 3 field sources are expected in the external annulus against 4 observed. There is a $2 \sigma$ excess

\footnotetext{
${ }^{1}$ Since the search radius $R_{\text {ext }}$ does not extend up to $1.0 \mathrm{Mpc}$ for all the clusters, the number of sources expected in each annulus was normalized by the area actually examined.
} 
Table 4. Radial source distribution for the brightest sources.

\begin{tabular}{lcccccc}
\hline \hline$R$ & $N_{0.5-2.0}$ & $\Sigma_{0.5-2.0}^{\mathrm{Cl}}$ & $\Sigma_{0.5-2.0}^{\text {field }}$ & $N_{2.0-10.0}$ & $\Sigma_{2.0-10.0}^{\mathrm{Cl}}$ & $\Sigma_{2.0-10.0}^{\text {field }}$ \\
$(1)$ & $(2)$ & $(3)$ & $(4)$ & $(5)$ & $(6)$ & $(7)$ \\
\hline $0-0.5$ & $7(2)$ & $0.50 \pm 0.19$ & $0.15 \pm 0.03$ & $7(2)$ & $0.50 \pm 0.19$ & $0.13 \pm 0.03$ \\
$0.5-1.0$ & $4(3)$ & $0.18 \pm 0.09$ & $0.15 \pm 0.03$ & $4(3)$ & $0.18 \pm 0.09$ & $0.13 \pm 0.03$ \\
\hline
\end{tabular}

- Column 1: size of the annulus in Mpc;

- Col. 2: number of sources detected in the annulus with an unabsorbed flux brighter than $1.25 \times 10^{-14} \mathrm{erg} \mathrm{cm}^{-2} \mathrm{~s}^{-1}$ in the soft energy band. In parentheses the expected number of field sources over the cluster regions is given;

- Col. 3: soft X-ray surface density for the cluster region sources in $\mathrm{Mpc}^{-2}$;

- Col. 4: estimated soft X-ray surface density for the control field sources in $\mathrm{Mpc}^{-2}$;

- Col. 5: number of sources detected in the circular annulus with a flux brighter than $2.5 \times 10^{-14} \mathrm{erg} \mathrm{cm}^{-2} \mathrm{~s}^{-1}$ in the hard energy band. In parentheses the expected number of field sources over the cluster regions is given;

- Col. 6: hard X-ray surface density for the cluster regions sources in $\mathrm{Mpc}^{-2}$;

- Col. 7: estimated hard X-ray surface density for the control field sources in $\mathrm{Mpc}^{-2}$.

within $0.5 \mathrm{Mpc}$ from the cluster center, thereby confirming the small excess of sources found in Sect. 6.1 and providing additional support for the possible existence of X-ray sources belonging to the cluster (on average one every three clusters).

Next we constructed the radial profile of the surface density, $\Sigma^{\mathrm{cl}}$, within $1 \mathrm{Mpc}$ (Cols. 3 and 6 of Table 4), assuming the brightest sources to be at the cluster redshift and taking the areas effectively surveyed into account. The field source number density per $\mathrm{Mpc}^{2}, \Sigma^{\text {field }}$ (Cols. 4 and 7), was then derived assuming the same redshift distribution as in our cluster sample.

The radial profile of the soft source surface density, $\Sigma^{\mathrm{cl}}$, was compared with the distribution of Ruderman \& Ebeling (2005) in a subsample of the MACS clusters. The MACS survey was built to find the "most" massive clusters ever (see Ebeling et al. 2001). The median MACS X-ray luminosity ${ }^{2}$ is 2.5 times our median luminosity with only 3 (out of 18) of our clusters more luminous than the MACS median luminosity. The flux limit we adopted in the soft band is the same as the one used by them $\left(1.25 \times 10^{-14} \mathrm{erg} \mathrm{cm}^{-2} \mathrm{~s}^{-1}\right)$. Within $0.5 \mathrm{Mpc}$, where the excess is found, the two densities are in very good agreement $\left(0.50\right.$ sources per $\mathrm{Mpc}^{2}$ from Table 4 vs. 0.53 sources per $\mathrm{Mpc}^{2}$ from their Fig. 2). Within $0.5 \mathrm{Mpc}$ the excess of Ruderman \& Ebeling (2005) is at $\approx 4 \sigma$ with respect to their point-source density at the cluster field edges (4-7 Mpc) that they assume to be the background point-source density ${ }^{3}$. Between 0.5 and $1 \mathrm{Mpc}$, the two profiles are consistent within the errors. We stress that the field surface density obtained from our control fields agree very well with the one derived by Ruderman \& Ebeling (2005) from their cluster field edges.

In order to increase the statistics, we extended our radial analysis to fainter sources. We used 31 sources with a soft unabsorbed flux brighter than $2.7 \times 10^{-15} \mathrm{erg} \mathrm{cm}^{-2} \mathrm{~s}^{-1}$ and 25 sources with a hard flux brighter than $0.8 \times 10^{-14} \mathrm{erg} \mathrm{cm}^{-2} \mathrm{~s}^{-1}$ since all clusters are complete down to these flux limits. A small source excess is found within $0.5 \mathrm{Mpc}$ from the clusters center, which however does not improve significantly the previous result, indicating that cluster X-ray sources are confined to the highest fluxes.

${ }^{2}$ The MACS median luminosity was estimated using Fig. 10 in Ebeling et al. (2001) after converting their 0.1-2.4 keV ROSAT luminosity into bolometric luminosity with their same assumptions on the temperature, and taking into account the different cosmologies assumed.

3 The significance of $8.0 \sigma$ claimed by Ruderman \& Ebeling (2005) is actually for the area within $3.5 \mathrm{Mpc}$ from the cluster centers.

\subsection{Optical counterparts}

We checked the literature for any optical counterpart associated with the $13 \mathrm{X}$-ray sources with a soft flux brighter than $1.25 \times 10^{-14} \mathrm{erg} \mathrm{cm}^{-2} \mathrm{~s}^{-1}$ and/or a hard flux brighter than $2.5 \times 10^{-14} \mathrm{erg} \mathrm{cm}^{-2} \mathrm{~s}^{-1}$. The results of this search are reported in Table 5. No optical data are available for four sources. Two sources are identified with cluster members with spectroscopic redshifts. Three are identified with optically faint objects with no redshift measurement. Finally three sources are background objects having a significantly higher redshift than those of the corresponding clusters. A fourth one may also be a background object on the basis of a photometric redshift. Clearly more optical work is needed in order to know the cluster membership of these objects.

\section{Comparison with radio sources in X-ray selected clusters}

As mentioned in the introduction, radio galaxies in both nearby and distant clusters have a centrally peaked distribution. Branchesi et al. (2006) analyzed a sample of VLA radio sources detected in 18 X-ray selected clusters (Gioia et al. 2001) extracted from the NEP survey with redshift and luminosity distributions similar to the present sample. They found a pronounced peak of radio sources within 0.2 Abell radii, corresponding (in the cosmology adopted in Branchesi et al. 2006) roughly to the $0.5 \mathrm{Mpc}$ size of the first bin in Table 4 here. The radio source surface density associated to this peak is $\approx 10 \mathrm{Mpc}^{-2}$, i.e. $\sim 20$ times higher than the X-ray point source surface density found here. However, the larger radio excess could be a selection effect due to the better sensitivity of the radio observations. Falcke et al. (2004) discuss a correlation between the 3-9 keV luminosity and the $5 \mathrm{GHz}$ core radio luminosity $\left(v L_{v}\right)$ in radioloud AGN. To estimate the X-ray luminosity expected for the Branchesi et al. (2006) radio galaxies, we first converted the total radio luminosity to the core radio luminosity using the correlation between the core and radio powers published by Giovannini et al. (1988). From the resulting core radio luminosity and the Falcke correlation, we find that the estimated X-ray luminosity of the NEP radio sources is about two orders of magnitude lower than the X-ray luminosity reached by the present Chandra observations. Thus the X-ray instruments do not seem to have the sensitivity required to detect such faint X-ray counterparts of the radio-loud AGN. Similar arguments apply if the weaker NEP radio sources are star-forming galaxies due to the tight linear 
Table 5. Optical parameters for the $13 \mathrm{X}$-ray brightest sources.

\begin{tabular}{|c|c|c|c|c|c|c|c|}
\hline Cluster name & \# & RA & Dec & $\Delta \mathrm{RA}(\mathrm{x}-\mathrm{O})$ & $\Delta \operatorname{Dec}(\mathrm{x}-\mathrm{O})$ & $z$ & Notes \\
\hline & (2) & hh $\operatorname{mm}_{(3)}$ ss.s & - ' & $\begin{array}{c}\mathrm{s} \\
(5)\end{array}$ & (6) & & \\
\hline$\frac{(1)}{\text { Ahell2125 }}$ & $\frac{(2)}{5}$ & & & & & & (8) \\
\hline Abell 2125 & & 154056.4 & +66 1628.7 & +0.0 & -0.3 & 1.012 & $\mathrm{a}$ \\
\hline ZW CL 0024+1652 & 2 & 002631.1 & +171017.3 & +0.0 & +0.7 & $0.400 *$ & b \\
\hline MS $1621+2640$ & 4 & 162343.7 & +263244.7 & -0.1 & -0.3 & - & $\mathrm{c}$ \\
\hline \multirow[t]{2}{*}{ CL $1641+4001$} & 2 & 164150.3 & +400145.7 & - & - & - & - \\
\hline & 3 & 164154.2 & +400032.6 & +0.0 & +0.6 & 1.003 & $\mathrm{~d}$ \\
\hline V $1524+0957$ & 7 & 152443.4 & +095536.0 & - & - & - & - \\
\hline V $1121+2327$ & 3 & 112054.0 & +232704.9 & - & - & - & - \\
\hline V $1221+4918$ & 3 & 122120.1 & +491844.0 & - & - & - & - \\
\hline MS $1137+6625$ & 4 & 114031.2 & +660858.2 & +0.0 & +0.0 & 1.269 & $\mathrm{e}$ \\
\hline RDCSJ 1350+6007 & 8 & 135057.7 & +600813.7 & +0.1 & -2.2 & - & $\mathrm{c}$ \\
\hline \multirow[t]{2}{*}{ RXJ $1716+6708$} & 1 & 171636.9 & +670830.0 & +0.0 & +1.0 & $0.795 *$ & $\mathrm{f}$ \\
\hline & 4 & 171651.7 & +670854.8 & -0.0 & -1.1 & - & $\mathrm{g}$ \\
\hline MS 1054-0321 & 3 & 105658.8 & -033851.2 & -0.1 & -0.2 & $1.200^{\mathrm{ph}}$ & $\mathrm{h}$ \\
\hline
\end{tabular}

- Column 1: cluster name;

- Col. 2: source identification number as in Table 2;

- Cols. 3-4: x-ray source position; Right Ascension and Declination (Equatorial J2000, HH MM SS.S, +DD MM SS) as in Table 2;

- Cols. 5-6: offset between the position of the X-ray source and that of the optical counterpart ( $\triangle \mathrm{RA}$ in seconds of time and $\triangle \mathrm{DEC}$ in arcsec);

- Col. 7: spectroscopic or photometric (indicated by ${ }^{\mathrm{ph}}$ ) redshift. Asterisk indicates cluster membership;

- Col. 8: literature source: a) Miller et al. (2004); b) Czoske et al. (2001); c) Eckart et al. (2005); d) from the Sloan Digital Sky Survey; e) Silverman et al. (2005); f) Gioia et al. (1999); g) from a Keck image taken by I. Gioia and h) (Förster Schreiber et al. 2006).

relations between the X-ray, radio, and far infrared luminosities found, among others, by Ranalli et al. (2003).

On the other hand, the dozen X-ray sources at the bright end of the $\log N-\log S$ have a high enough X-ray luminosity to statistically expect that radio emission from some of them would be detectable in surveys like the FIRST (Becker et al. 1995) or the NVSS (NRAO VLA Sky Survey, Condon et al. 1998). Indeed two such X-ray sources have associated radio emission. Source \# 3 in CL $1641+4001$ has a FIRST counterpart at the position $16^{\mathrm{h}} 41^{\mathrm{m}} 54.24^{\mathrm{s}},+40^{\circ} 00^{\prime} 32.0^{\prime \prime}$ with a flux $S_{1.4 \mathrm{GHz}}=5.06 \mathrm{mJy}$. Source \# 1 in RXJ 1716.4+6708 has a NVSS counterpart at the position $17^{\mathrm{h}} 16^{\mathrm{m}} 37.14^{\mathrm{s}}, 67^{\circ} 08^{\prime} 28.8^{\prime \prime}$ with a flux $S_{1.4 \mathrm{GHz}}=332.0 \mathrm{mJy}(4 \mathrm{C}+67.26)$. Given the low statistics no conclusion is drawn here.

For completeness the coordinates of all sources listed in Table 2 were cross-correlated with those of the FIRST (or NVSS catalogs when no FIRST data are available). Only one additional coincidence was found for source \# 2 in ZW CL 1454.8+2233. The corresponding FIRST radio source at $14^{\mathrm{h}} 57^{\mathrm{m}} 10.82^{\mathrm{s}},+22^{\circ} 18^{\prime} 44.9^{\prime \prime}$ has a flux of $S_{1.4 \mathrm{GHz}}=4.87 \mathrm{mJy}$.

\section{Summary and conclusions}

In this paper we have presented an analysis of the X-ray point sources detected in the inner $1 \mathrm{Mpc}$ region of 18 high- $z(0.25<$ $z<1.01)$ galaxy clusters retrieved from the Chandra archive. Unlike most of the previous studies that analyzed the whole Chandra field around the clusters, we considered only the point sources embedded in the cluster emission, i.e. belonging to the clusters or in projection. We find a small excess for the cluster sources at fluxes brighter than $1.25 \times 10^{-14} \mathrm{erg} \mathrm{cm}^{-2} \mathrm{~s}^{-1}$ in the soft energy band and brighter than $2.5 \times 10^{-14} \mathrm{erg} \mathrm{cm}^{-2} \mathrm{~s}^{-1}$ in the hard energy band. The significance of the excess is at the $1.7 \sigma$ level in each band (Sect. 6.1). To further investigate the reality of the X-ray point source excess, we examined the source radial distribution as a function of the projected linear distance from the cluster center. A $2 \sigma$ excess was found within $0.5 \mathrm{Mpc}$ providing additional support for the existence of X-ray sources belonging to the cluster. The excess is given by $\approx 6$ out of 11 sources in the luminosity range $3 \times 10^{42}-10^{44} \mathrm{erg} \mathrm{s}^{-1}$ in the soft and $10^{43}-10^{44} \mathrm{erg} \mathrm{s}^{-1}$ in the hard energy band. These results agree with those of previous studies that have detected excesses in cluster fields.

The galaxy clusters presented here are in the bolometric luminosity range $10^{44}-\sim 5 \times 10^{45} \mathrm{erg} \mathrm{s}^{-1}$, with a median value of $0.9 \times 10^{45} \mathrm{erg} \mathrm{s}^{-1}$ (only three clusters have a luminosity higher than $2 \times 10^{45} \mathrm{erg} \mathrm{s}^{-1}$ ). Ruderman \& Ebeling (2005) examined a sample of 51 clusters in a similar redshift range $(0.3<z<0.7)$, but using more massive clusters than ours. They found a soft excess within $1 \mathrm{Mpc}$ similar to ours. Jeltema et al. (2006) analyzed six groups of galaxies in the redshift range $0.2<z<0.6$ and obtained a $2 \sigma$ overdensity result for these lower-mass systems. Therefore our results provide further evidence of the presence of a population of AGN in systems with very different masses. While Ruderman \& Ebeling (2005) did not analyze sources in the hard band, Jeltema et al. (2006) found no significant excess in the number of hard sources. Thus our study provides for the first time some evidence of source overdensity within $1 \mathrm{Mpc}$ in both energy bands. In addition, there is a slight indication that the hard band excess increases with redshift, even though the small statistics do not allow us to draw any conclusion. We note, however, that this effect goes in the same direction as the apparent correlation between the amplitude of the overdensity and the cluster redshift found for the first time by Cappelluti et al. (2005) (see also Martini et al. 2006).

Radio galaxies in both nearby and distant clusters have a centrally peaked distribution with surface density within $0.5 \mathrm{Mpc}$ on the order of $10 \mathrm{Mpc}^{-2}$. A recent work by Branchesi et al. (2006) on a sample of distant X-ray selected clusters $(0.3<z<0.8)$ finds an excess in the radio surface density within $0.5 \mathrm{Mpc}$ from the cluster center that is much higher (by a factor $\sim 20$ ) than the present X-ray source overdensity. Even if an excess of sources is present at both wavelengths, the much smaller amplitude of the X-ray overdensity could be explained by the better sensitivity of the radio observations. Higher sensitivity (and resolution) X-ray telescopes could provide more information on the nature of the 
population of AGN and/or star-forming galaxies in high- $z$ clusters, which is responsible for the more pronounced excess in the radio domain.

Acknowledgements. We acknowledge stimulating discussions with Paolo Tozzi, Anna Wolter and Mauro Dadina. An anonymous referee gave substantial comments which helped to improve this paper. Partial financial support for this work came from the Italian Space Agency ASI (Agenzia Spaziale Italiana) through grant ASI-INAF I/023/05/0. This research made use of data obtained from the Chandra Data Archive (CDA), which is part of the Chandra X-Ray Observatory Science Center, operated for the National Aeronautics and Space Administration (NASA) by the Smithsonian Astrophysical Observatory (SAO).

\section{References}

Becker, R. H., White, R. L., \& Helfand, D. J. 1995, ApJ, 450, 559

Branchesi, M., Gioia, I. M., Fanti, C., Fanti, R., \& Perley, R. 2006, A\&A, 446, 97

Brandt, W. N., Alexander, D. M., Hornschemeier, A. E., et al. 2001, AJ, 122, 2810

Butcher, H., \& Oemler, A. 1978, ApJ, 219, 18

Butcher, H., \& Oemler, A. 1984, ApJ, 285, 426

Cappelluti, N., Cappi, M., Dadina, M., et al. 2005, A\&A, 430, 39

Cappelluti, N., Böringer, H., Schuecker, P., et al. 2007, A\&A, submitted

Cappi, M., Mazzotta, P., Elvis, M., et al. 2001, ApJ, 548, 624

Condon, J. J., Cotton, W. D., Greisen, E. W., et al. 1998, AJ, 115, 1693

Crawford, D. F., Jauncey, D. L., \& Murdoch, H. S. 1970, ApJ, 162, 405

Czoske, O., Kneib, J.-P., Soucail, G., et al. 2001, A\&A, 372, 391

D'Elia, V., Fiore, F., Elvis, M., et al. 2004, A\&A, 422, 11

Dickey, J. M., \& Lockman, F. J. 1990, ARA\&A, 28, 215

Dressler, A., Thompson, I. B., \& Shectman, S. A. 1985, ApJ, 288, 481

Dressler, A., Smail, I., Poggianti, et al. 1999, ApJS, 122, 51

Ebeling, H., Edge, A. C., \& Henry, J. P. 2001, ApJ, 553, 668

Eckart, M. E., Laird, E. S., Stern, D., et al. 2005, ApJS, 156, 35

Falcke, H., Körding, E., \& Markoff, S. 2004, A\&A, 414, 895

Fanti, R. 1984, Clusters and Groups of Galaxies, ASSL, 111, 185

Förster Schreiber, N. M., Franx, M., Labbé, I., et al. 2006, AJ, 131, 1891

Freeman, P. E., Kashyap, V., Rosner, R., \& Lamb, D. Q. 2002, ApJS, 138, 185

Gehrels, N. 1986, ApJ, 303, 336

Giacconi, R., Rosati, P., Tozzi, P., et al. 2001, ApJ, 551, 624

Gioia, I. M., Maccacaro, T., Schild, R. E., et al. 1990, ApJS, 72, 567

Gioia, I. M., Henry, J. P., Mullis, C. R., Ebeling, H., \& Wolter, A. 1999, AJ, 117, 2608

Gioia, I. M., Henry, J. P., Mullis, C. R., et al. 2001, ApJ, 553, L105

Gioia, I. M., Henry, J. P., Mullis, C. R., et al. 2003, ApJS, 149, 29

Giovannini, G., Feretti, L., Gregorini, L., \& Parma, P. 1988, A\&A, 199, 73

Grant, C. E., Bautz, M. W., Kissel, S. M., LaMarr, B., \& Prigozhin, G. Y. 2005, Proc. SPIE, 5898, 201
Hasinger, G., Burg, R., Giacconi, R., et al. 1998, A\&A, 329, 482 Henry, J. P., \& Briel, U. G. 1991, A\&A, 246, L14

Henry, J. P., Mullis, C. R., Voges, W., et al. 2006, ApJS, 162, 304

Jeltema, T. E., Mulchaey, J. S., Lubin, L. M., Rosati, P., \& Böhringer, H. 2006, ApJ, 649, 649

Johnson, O., Best, P. N., \& Almaini, O. 2003, MNRAS, 343, 924

Lazzati, D., Campana, S., Rosati, P., Chincarini, G., \& Giacconi, R. 1998, A\&A, 331,41

Ledlow, M. J., \& Owen, F. N. 1995a, AJ, 109, 853

Ledlow, M. J., \& Owen, F. N. 1995b, AJ, 110, 1959

Ledlow, M. J., \& Owen, F. N. 1996, AJ, 112, 9

Manners, J. C., Johnson, O., Almaini, O., et al. 2003, MNRAS, 343, 293

Markevitch, M. 2001, CXC ACIS background memo (Cambridge: CXC)

Markevitch, M., Bautz, M. W., Biller, B., et al. 2003, ApJ, 583, 70

Marshall, H. L., et al. 2003, Chandra Calibration Workshop (Cambridge: CXC), http://cxc.harvard.edu/ccw/proceedings/03_proc/ presentations/marshall2

Martini, P., Kelson, D. D., Mulchaey, J. S., \& Trager, S. C. 2002, ApJ, 576, L109

Martini, P., Kelson, D. D., Kim, E., Mulchaey, J. S., \& Athey, A. A. 2006, ApJ, 644, 116

Miller, N. A., Owen, F. N., Hill, J. M., et al. 2004, ApJ, 613, 841

Molnar, S. M., Hughes, J. P., Donahue, M., \& Joy, M. 2002, ApJ, 573, L91

Mullis, C. R., McNamara, B. R., Quintana, H., et al. 2003, ApJ, 594, 154

Murdoch, H. S., Crawford, D. F., \& Jauncey, D. L. 1973, ApJ, 183, 1

Mushotzky, R. F. 1984, Adv. Space Res., 3, 157

Mushotzky, R. F., Cowie, L. L., Barger, A. J., \& Arnaud, K. A. 2000, Nature, 404, 459

Owen, F. N. 1975, ApJ, 195, 593

Pentericci, L., Kurk, J. D., Carilli, C. L., et al. 2002, A\&A, 396, 109

Perlman, E. S., Horner, D. J., Jones, L. R., et al. 2002, ApJS, 140, 265

Plucinsky, P. P., \& Virani, S. N. 2000, Proc. SPIE, 4012, 681

Ranalli, P., Comastri, A., \& Setti, G. 2003, A\&A, 399, 39

Rosati, P., Della Ceca, R., Norman, C., \& Giacconi, R. 1998, ApJ, 492, L21

Rosati, P., Giacconi, R., Gilli, R., et al. 2002, ApJ, 566, 667

Ruderman, J. T., \& Ebeling, H. 2005, ApJ, 623, L81

Silverman, J. D., Green, P. J., Barkhouse, W. A., et al. 2005, ApJ, 618, 123

Spergel, D. N., Verde, L., Peiris, H. V., et al. 2003, ApJS, 148, 175

Strüder, L., Briel, U., Dennerl, K., et al. 2001, A\&A, 365, L18

Sun, M., \& Murray, S. S. 2002, ApJ, 577, 139

Townsley, L. K., Broos, P. S., Garmire, G. P., \& Nousek, J. A. 2000, ApJ, 534, L139

Tozzi, P., Rosati, P., Nonino, M., et al. 2001, ApJ, 562, 42

Turner, M. J. L., Abbey, A., Arnaud, M., et al. 2001, A\&A, 365, L27

van Speybroeck, L. P., Jerius, D., Edgar, R. J., et al. 1997, Proc. SPIE, 3113, 89

Vikhlinin, A., McNamara, B. R., Forman, W., et al. 1998, ApJ, 502, 558

Vikhlinin, A., Markevitch, M., Murray, S. S., et al. 2005, ApJ, 628, 655

Voges, W., Aschenbach, B., Boller, Th., et al. 1999, A\&A, 349, 389

Wang, J. X., Malhotra, S., Rhoads, J. E., et al. 2004, AJ, 127, 213

Wilkes, B. J., \& Elvis, M. 1987, ApJ, 323, 243 
M. Branchesi et al.: X-ray point source counts in areas covered by distant clusters, Online Material p 1

\section{Online Material}


M. Branchesi et al.: X-ray point source counts in areas covered by distant clusters, Online Material p 2

Table 2. X-ray cluster region point sources.

\begin{tabular}{|c|c|c|c|c|c|c|c|c|c|}
\hline Cluster name & $\begin{array}{l}\begin{array}{l}R_{\text {ext }} \\
" \\
(2)\end{array} \\
\text { (2) }\end{array}$ & (3) & $\begin{array}{c}\text { RA } \\
\text { hh mm ss.s } \\
\text { (4) }\end{array}$ & $\begin{array}{c}\text { Dec } \\
{ }^{\prime} \prime \prime \\
(5)\end{array}$ & $C_{0.5-2.0}$ & $C_{2.0-7.0}$ & $\begin{array}{r}S_{0.5-2.0} \\
10^{-15} \mathrm{cgs} \\
(8)\end{array}$ & $c_{N_{\mathrm{H}}}$ & $\begin{array}{r}S_{2.0-10.0} \\
10^{-15} \mathrm{cgs} \\
(10)\end{array}$ \\
\hline \multirow[t]{25}{*}{ Abell 2125} & 241 & 1 & 154039.4 & +661713.2 & 30.63 & 10.77 & $2.28 \pm 0.43$ & 1.083 & $3.49 \pm 1.13$ \\
\hline & & 2 & 154045.3 & +661727.3 & 9.77 & 7.75 & $0.73 \pm 0.24$ & & $2.52 \pm 0.96$ \\
\hline & & 3 & 154046.7 & +66 1321.0 & 11.92 & & $0.84 \pm 0.24$ & & \\
\hline & & 4 & 154052.4 & +661236.9 & 104.72 & 39.61 & $7.28 \pm 0.74$ & & $12.50 \pm 2.38$ \\
\hline & & 5 & 154056.4 & +661628.7 & 420.99 & 141.58 & $30.96 \pm 1.76$ & & $45.71 \pm 6.11$ \\
\hline & & 6 & 154058.9 & +661742.8 & 13.82 & & $1.05 \pm 0.30$ & & \\
\hline & & 7 & 154100.4 & +661903.0 & 13.83 & & $1.11 \pm 0.31$ & & \\
\hline & & 8 & 154102.0 & +661721.4 & 82.37 & 27.88 & $6.25 \pm 0.71$ & & $9.17 \pm 2.01$ \\
\hline & & 9 & 154102.0 & +661627.2 & 27.31 & & $2.02 \pm 0.40$ & & \\
\hline & & 10 & 154102.7 & +661404.7 & 36.60 & 15.70 & $2.62 \pm 0.44$ & & $5.01 \pm 1.38$ \\
\hline & & 11 & 154109.2 & +661449.0 & 8.55 & & $0.62 \pm 0.22$ & & \\
\hline & & 12 & 154109.8 & +661545.3 & 10.37 & & $0.77 \pm 0.26$ & & \\
\hline & & 13 & 154112.4 & +661717.1 & 18.37 & 9.19 & $1.42 \pm 0.35$ & & $3.06 \pm 1.10$ \\
\hline & & 14 & 154116.9 & +661626.9 & 10.14 & & $0.78 \pm 0.27$ & & \\
\hline & & 15 & 154117.4 & +661924.0 & 21.02 & & $1.76 \pm 0.40$ & & \\
\hline & & 16 & 154117.8 & +661343.1 & 19.59 & 12.72 & $1.45 \pm 0.33$ & & $4.18 \pm 1.26$ \\
\hline & & 17 & 154126.2 & +661341.4 & & 6.73 & & & $2.36 \pm 0.96$ \\
\hline & & 18 & 154127.3 & +66 1741.5 & 14.39 & & $1.40 \pm 0.39$ & & \\
\hline & & 19 & 154127.4 & +661413.7 & 19.64 & 16.55 & $1.77 \pm 0.41$ & & $6.51 \pm 1.76$ \\
\hline & & 20 & 154128.3 & +66 1247.5 & 6.84 & & $0.60 \pm 0.23$ & & \\
\hline & & 21 & 154133.7 & +661342.1 & 16.58 & 7.45 & $1.30 \pm 0.32$ & & $2.54 \pm 1.00$ \\
\hline & & 22 & 154137.3 & +661507.1 & 9.34 & & $0.75 \pm 0.25$ & & \\
\hline & & 23 & 154141.1 & +661642.0 & 8.41 & & $0.70 \pm 0.25$ & & \\
\hline & & 24 & 154143.5 & +661419.4 & 15.76 & & $1.28 \pm 0.34$ & & \\
\hline & & 25 & 154145.0 & +661510.7 & & 9.61 & & & $3.47 \pm 1.25$ \\
\hline \multirow[t]{7}{*}{ ZW CL 1454+2233 } & 200 & 1 & 14579.7 & +222304.0 & 22.10 & 14.17 & $1.69 \pm 0.40$ & 1.096 & $4.29 \pm 1.32$ \\
\hline & & 2 & 145710.8 & +221845.0 & 46.94 & 21.56 & $3.13 \pm 0.47$ & & $6.11 \pm 1.47$ \\
\hline & & 3 & 145712.2 & +222142.4 & 87.63 & 27.32 & $6.41 \pm 0.76$ & & $8.10 \pm 1.90$ \\
\hline & & 4 & 145713.2 & +22 1727.0 & 25.65 & 15.59 & $1.71 \pm 0.34$ & & $4.43 \pm 1.23$ \\
\hline & & 5 & 145714.8 & +221933.5 & 29.82 & & $2.07 \pm 0.47$ & & \\
\hline & & 6 & 145717.7 & +221922.8 & 35.03 & 17.83 & $2.58 \pm 0.48$ & & $5.46 \pm 1.48$ \\
\hline & & 7 & 145721.0 & +222335.3 & 88.99 & 22.61 & $7.44 \pm 0.89$ & & $7.15 \pm 1.86$ \\
\hline \multirow[t]{10}{*}{ MS 1008-1224 } & 172 & 1 & 101021.4 & -124007.9 & $5.90 *$ & 7.84 & $0.69 \pm 0.29$ & 1.203 & $4.49 \pm 1.69$ \\
\hline & & 2 & 101024.7 & -124016.9 & & 13.74 & & & $8.81 \pm 2.57$ \\
\hline & & 3 & 101026.4 & -123810.9 & 7.50 & & $0.92 \pm 0.35$ & & \\
\hline & & 4 & 101029.0 & -124013.5 & & 25.93 & & & $15.39 \pm 3.47$ \\
\hline & & 5 & 101032.3 & -123934.8 & 17.68 & & $2.21 \pm 0.66$ & & \\
\hline & & 6 & 101035.3 & -124022.0 & 47.40 & 16.31 & $6.45 \pm 0.97$ & & $10.67 \pm 2.91$ \\
\hline & & 7 & 101037.1 & -123857.8 & 16.82 & & $2.47 \pm 0.66$ & & \\
\hline & & 8 & 101039.4 & -124109.2 & 9.40 & & $1.24 \pm 0.42$ & & \\
\hline & & 9 & 101042.7 & -123919.1 & 20.88 & & $2.84 \pm 0.65$ & & \\
\hline & & 10 & 101041.8 & -124002.3 & 11.84 & & $1.59 \pm 0.50$ & & \\
\hline \multirow[t]{6}{*}{ ZW CL $0024+1652$} & 118 & 1 & 002631.0 & +171030.4 & 15.71 & & $1.34 \pm 0.34$ & 1.125 & \\
\hline & & 2 & 002631.1 & +171017.3 & 235.11 & 66.39 & $20.81 \pm 1.38$ & & $43.79 \pm 7.25$ \\
\hline & & 3 & 002631.7 & +171022.6 & 16.70 & & $1.48 \pm 0.36$ & & \\
\hline & & 4 & 002632.0 & +170941.9 & & 19.60 & & & $12.52 \pm 3.17$ \\
\hline & & 5 & 002633.0 & +170759.9 & 33.51 & 6.70 & $2.93 \pm 0.51$ & & $4.38 \pm 1.80$ \\
\hline & & 6 & 002633.3 & +171034.5 & & 6.74 & & & $4.27 \pm 1.74$ \\
\hline \multirow[t]{5}{*}{ MS 1621+2640 } & 148 & 1 & 162329.0 & +263446.8 & $5.81 *$ & & $1.01 \pm 0.43$ & 1.107 & \\
\hline & & 2 & 162333.9 & +263524.9 & 7.65 & 7.76 & $1.32 \pm 0.49$ & & $6.45 \pm 2.45$ \\
\hline & & 3 & 162340.3 & +263550.0 & & 7.77 & & & $6.58 \pm 2.49$ \\
\hline & & 4 & 162343.7 & +263244.7 & 282.84 & 107.61 & $52.80 \pm 3.37$ & & $94.81 \pm 13.50$ \\
\hline & & 5 & 162345.8 & +263335.2 & & 15.34 & & & $13.44 \pm 3.77$ \\
\hline \multirow[t]{3}{*}{ RXJ 1701+6414 } & 108 & 1 & 170113.1 & +641250.4 & $5.88^{*}$ & 7.61 & $0.67 \pm 0.28$ & 1.077 & $3.94 \pm 1.52$ \\
\hline & & 2 & 170121.5 & +641505.2 & & 8.54 & & & $4.50 \pm 1.65$ \\
\hline & & 3 & 170128.3 & +641332.7 & & 32.31 & & & $16.54 \pm 3.40$ \\
\hline \multirow[t]{6}{*}{ CL 1641+4001 } & 89 & 1 & 164150.0 & +400249.4 & 13.82 & 7.79 & $1.99 \pm 0.54$ & 1.030 & $4.57 \pm 1.72$ \\
\hline & & 2 & 164150.3 & +400145.7 & 162.94 & 46.56 & $22.74 \pm 1.95$ & & $26.83 \pm 4.83$ \\
\hline & & 3 & 164154.2 & +400032.6 & 172.48 & 93.56 & $23.72 \pm 1.98$ & & $53.58 \pm 7.85$ \\
\hline & & 4 & 164153.6 & +400145.0 & 8.71 & & $1.22 \pm 0.45$ & & \\
\hline & & 5 & 164155.4 & +400143.0 & & 7.59 & & & $4.38 \pm 1.70$ \\
\hline & & 6 & 164158.4 & +400048.4 & 9.89 & 6.89 & $1.38 \pm 0.44$ & & $3.98 \pm 1.58$ \\
\hline \multirow[t]{2}{*}{ V 1524+0957 } & 148 & 1 & 152430.6 & +095730.5 & 12.77 & 13.76 & $1.63 \pm 0.46$ & 1.087 & $7.20 \pm 2.09$ \\
\hline & & 2 & 152432.3 & +095745.1 & 23.74 & 18.80 & $2.97 \pm 0.62$ & & $9.75 \pm 2.48$ \\
\hline
\end{tabular}


Table 2. continued.

\begin{tabular}{|c|c|c|c|c|c|c|c|c|c|}
\hline Cluster name & $\begin{array}{l}R_{\text {ext }} \\
\text { " } \\
\text { (2) }\end{array}$ & (3) & $\begin{array}{c}\text { RA } \\
\text { hh mm ss.s } \\
\text { (4) }\end{array}$ & $\begin{array}{c}\text { Dec } \\
\text { ', } \\
(5)\end{array}$ & $\begin{array}{r}C_{0.5-2.0} \\
(6)\end{array}$ & $C_{2.0-7.0}$ & $\begin{array}{r}S_{0.5-2.0} \\
10^{-15} \text { cgs } \\
(8)\end{array}$ & $c_{N_{\mathrm{H}}}$ & $\begin{array}{r}S_{2.0-10.0} \\
10^{-15} \mathrm{cgs} \\
(10)\end{array}$ \\
\hline \multirow{6}{*}{ (1) } & & 3 & 152432.4 & +095907.2 & & 5.91 & & & $3.39 \pm 1.45$ \\
\hline & & 4 & 152435.5 & +095822.3 & 7.82 & 9.74 & $0.99 \pm 0.36$ & & $5.24 \pm 1.79$ \\
\hline & & 5 & 152438.0 & +095853.2 & 21.56 & 10.81 & $2.55 \pm 0.56$ & & $5.45 \pm 1.77$ \\
\hline & & 6 & 152442.4 & +100001.0 & 6.89 & 6.83 & $0.85 \pm 0.33$ & & $3.69 \pm 1.48$ \\
\hline & & 7 & 152443.4 & +095536.0 & 221.31 & 95.38 & $28.86 \pm 0.22$ & & $50.83 \pm 7.45$ \\
\hline & & 8 & 152443.7 & +095605.1 & 7.55 & & $0.96 \pm 0.36$ & & \\
\hline \multirow[t]{4}{*}{ MS 0451.6-0305 } & 148 & 1 & 045412.9 & -030046.8 & 42.92 & & $3.33 \pm 0.58$ & 1.156 & \\
\hline & & 2 & 045410.9 & -03 0124.3 & 13.26 & & $1.13 \pm 0.35$ & & \\
\hline & & 3 & 045412.3 & -025911.3 & 6.74 & 7.78 & $0.54 \pm 0.21$ & & $4.47 \pm 1.70$ \\
\hline & & 4 & 045416.0 & -030232.2 & & 6.76 & & & $4.01 \pm 1.64$ \\
\hline \multirow[t]{5}{*}{ V1121+2327 } & 128 & 1 & 112049.7 & +232721.2 & 54.77 & 26.67 & $4.70 \pm 0.65$ & 1.039 & $10.10 \pm 2.23$ \\
\hline & & 2 & 112049.8 & +232630.4 & 8.83 & 13.83 & $0.75 \pm 0.25$ & & $5.17 \pm 1.50$ \\
\hline & & 3 & 112054.0 & +232704.9 & 160.70 & 44.51 & $13.98 \pm 1.19$ & & $16.89 \pm 3.09$ \\
\hline & & 4 & 112058.8 & +232629.6 & & 8.48 & & & $3.25 \pm 1.20$ \\
\hline & & 5 & 112104.7 & +232511.4 & & 6.78 & & & $2.64 \pm 1.07$ \\
\hline \multirow[t]{2}{*}{ MS 2053-0449 } & 118 & 1 & 205614.3 & -043716.8 & 9.81 & & $1.36 \pm 0.44$ & 1.149 & \\
\hline & & 2 & 205618.7 & -043914.6 & 7.91 & & $1.03 \pm 0.37$ & & \\
\hline \multirow[t]{6}{*}{ V1221+4918 } & 143 & 1 & 122112.6 & +491919.1 & 6.68 & & $0.52 \pm 0.21$ & 1.043 & \\
\hline & & 2 & 122118.1 & +49 1635.6 & & 7.82 & & & $2.47 \pm 0.93$ \\
\hline & & 3 & 122120.1 & +49 1844.0 & 40.51 & 153.94 & $31.03 \pm 1.83$ & & $50.08 \pm 6.59$ \\
\hline & & 4 & 122126.3 & +491804.1 & & 12.18 & & & $3.92 \pm 1.23$ \\
\hline & & 5 & 122130.9 & +49 1757.3 & 9.29 & & $0.76 \pm 0.26$ & & \\
\hline & & 6 & 122129.1 & +491643.4 & 7.82 & 8.72 & $0.57 \pm 0.21$ & & $2.76 \pm 9.92$ \\
\hline \multirow[t]{5}{*}{ MS 1137+6625 } & 103 & 1 & 114006.2 & +660818.2 & 6.99 & & $0.30 \pm 0.12$ & 1.035 & \\
\hline & & 2 & 114012.8 & +660733.0 & 9.64 & 21.33 & $0.41 \pm 0.14$ & & $4.70 \pm 1.15$ \\
\hline & & 3 & 114020.4 & +660730.5 & 39.55 & 22.19 & $1.67 \pm 0.27$ & & $4.87 \pm 1.18$ \\
\hline & & 4 & 114031.2 & +660858.2 & 860.74 & 263.18 & $36.44 \pm 1.43$ & & $59.46 \pm 7.50$ \\
\hline & & 5 & 114033.7 & +660739.6 & 7.71 & 19.63 & $0.32 \pm 1.18$ & & $4.35 \pm 1.10$ \\
\hline \multirow[t]{4}{*}{ RDCSJ 1317+2911 } & 69 & 1 & 131718.9 & +291111.1 & 62.43 & 24.59 & $3.22 \pm 0.42$ & 1.031 & $5.54 \pm 1.27$ \\
\hline & & 2 & 131720.7 & +291201.6 & 6.85 & & $0.35 \pm 0.15$ & & \\
\hline & & 3 & 131722.0 & +291124.2 & & 21.58 & & & $4.89 \pm 1.18$ \\
\hline & & 4 & 131723.5 & +291149.5 & 7.70 & & $0.41 \pm 0.15$ & & \\
\hline \multirow[t]{9}{*}{ RDCSJ $1350+6007$} & 128 & 1 & 135037.8 & +600821.2 & 39.73 & 13.78 & $4.15 \pm 0.67$ & 1.052 & $6.11 \pm 1.78$ \\
\hline & & 2 & 135039.8 & +600506.8 & & 6.85 & & & $2.95 \pm 1.18$ \\
\hline & & 3 & 135043.0 & +600609.3 & 6.92 & & $0.68 \pm 0.26$ & & \\
\hline & & 4 & 135046.1 & +600658.2 & & 11.65 & & & $5.03 \pm 1.58$ \\
\hline & & 5 & 135050.2 & +600801.5 & 7.86 & & $0.98 \pm 0.35$ & & \\
\hline & & 6 & 135050.4 & +600620.5 & 6.72 & & $0.67 \pm 0.26$ & & \\
\hline & & 7 & 135057.0 & +600728.6 & & 9.79 & & & $4.59 \pm 1.56$ \\
\hline & & 8 & 135057.7 & +600813.7 & 123.40 & 21.74 & $13.04 \pm 1.25$ & & $9.63 \pm 2.31$ \\
\hline & & 9 & 135104.6 & +600627.5 & 11.89 & 10.86 & $1.22 \pm 0.37$ & & $4.76 \pm 1.54$ \\
\hline \multirow[t]{6}{*}{ RXJ 1716+6708 } & 108 & 1 & 171636.9 & +670830.0 & 8.71 & 55.42 & $1.05 \pm 0.36$ & 1.111 & $32.1 \pm 5.48$ \\
\hline & & 2 & 171637.6 & +670731.0 & 8.85 & & $1.02 \pm 0.34$ & & \\
\hline & & 3 & 171642.2 & +670659.8 & 17.33 & & $1.84 \pm 0.45$ & & \\
\hline & & 4 & 171651.7 & +670854.8 & 55.57 & 54.10 & $5.63 \pm 0.77$ & & $26.71 \pm 4.60$ \\
\hline & & 5 & 171653.1 & +670750.2 & 46.09 & 16.52 & $4.75 \pm 0.71$ & & $8.28 \pm 2.24$ \\
\hline & & 6 & 171707.4 & +670840.0 & & $5.85^{*}$ & & & $2.88 \pm 1.24$ \\
\hline \multirow[t]{4}{*}{ MS 1054-0321 } & 128 & 1 & 105651.4 & -033800.7 & 7.77 & & $0.35 \pm 0.13$ & 1.110 & \\
\hline & & 2 & 105652.6 & -033819.8 & 23.59 & 7.75 & $1.06 \pm 0.22$ & & $2.65 \pm 1.01$ \\
\hline & & 3 & 105658.8 & -033851.2 & 272.10 & 124.91 & $12.29 \pm 0.77$ & & $42.08 \pm 6.08$ \\
\hline & & 4 & 105704.9 & -03 3821.2 & & 9.71 & & & $3.31 \pm 1.14$ \\
\hline \multirow[t]{4}{*}{ WARPJ $1415+3612$} & 79 & 1 & 141511.9 & +361124.7 & 16.59 & 10.72 & $1.11 \pm 0.28$ & 1.033 & $3.03 \pm 0.99$ \\
\hline & & 2 & 141512.4 & +361303.9 & 8.70 & & $0.60 \pm 0.21$ & & \\
\hline & & 3 & 141513.5 & +36 1210.4 & & 22.51 & & & $6.39 \pm 1.52$ \\
\hline & & 4 & 141516.1 & +361151.8 & 40.76 & 19.70 & $2.77 \pm 0.45$ & & $5.62 \pm 1.40$ \\
\hline
\end{tabular}




\section{Appendix A: Data reduction}

We retrieved the level $=1$ event files from the archive and applied the standard processing. The CIAO tool acis_process_events was used to apply the correction for charge transfer inefficiency (CTI Townsley et al. 2000; Grant et al. 2005) and recomputation of event grades. To compute calibrated photon energies, acis_process_events was also used to update the Advanced CCD Imaging Spectrometer (ACIS) gain maps with the latest version provided within CALDB (ver. 3.0.3) and to correct for its time dependence (T_GAIN correction). The CTI and T_GAIN corrections were applied for those chips and period observations for which they were available. Most of the observations were telemetered in VFAINT mode, which provides a better rejection of the particle-induced background. For these observations we ran acis_process_events with the option "check_vf_pha = yes", to flag probable background events. After running acis_process_events, the events were filtered to include only the standard event grades $0,2,3,4$, and 6 and status bits set to 0 . In this way we removed photons detected in bad CCD columns and bad pixels, "problem" events such as cosmic ray afterglows, and also those with bad ASCA grades $(1,5$, and 7$)$.

The final step was to examine the background light curves during each observation to detect and remove the flaring episodes. After excluding the point sources and cluster emission from the event file, the script $l c \_c l e a n$ was used to extract and bin the light curve and to calculate the average count rate. The flare detection was performed following the recommendations on the energy band and the bin size given in the Markevitch $\mathrm{COOKBOOK}^{4}$. We excluded those time periods when the count rate was not within $20 \%$ of the quiescent rate. The final count rate in the source-free regions of each observation was compared with the background value tabulated by Markevitch. We obtained values consistent within less than $10 \%$. Compared to the ACIS front-side illuminated (FI) chips the back-side illuminated $(\mathrm{BI})$ chips (S1 and S3) have a higher sensitivity at low energies. However, this low-energy sensitivity makes the chip more sensitive to particle events, which results in more frequent background flares than in the FI chips (Plucinsky \& Virani 2000; Markevitch 2001; Markevitch et al. 2003). When the source lay in the S3 chip, the S1 chip was accurately examined to exclude completely the flare-affected period.

The released calibration underestimates the effective area of the Chandra mirror by $10 \%$ just above the Ir M edge, probably because the mirror surface is contaminated by a thin hydrocarbon layer (Marshall et al. 2003). To correct the effective area, a "positive absorption edge" described by Vikhlinin et al. (2005) was used in the analysis of the spectra of point sources with the $\mathrm{X}$-Ray Spectral Fitting Package (XSPEC).

\section{Appendix B: Computation of source parameters}

The net counts, $C$, calculated as the sum of all counts in the "source cell" subtracted by the sum of the background counts, $\mathrm{B}$, were used to estimate the source flux. Since the majority of the detected sources have poor statistics (less than 50 total net counts in the soft or the hard band), an estimate of the source flux through a fit to the data with an absorbed power law is not always possible. Two separate conversion factors, one for each band,

4 See http://cxc.harvard.edu/contrib/maxim/acisbg/ data/README and

http://cxc.harvard. edu/contrib/maxim/acisbg/COOKBOOK were then calculated to derive the flux $(S)$ from the observed count rate.

The net count rate for each source was computed by dividing the net counts by the effective exposure time at each source position. For each source, the effective exposure time is given by the observation exposure time (corrected for the flares' time periods) multiplied by the ratio of the exposure map, averaged within the extraction region for each source to the value of the exposure map at the aimpoint. The vignetting correction $(V)$ to be applied to the net counts is given by the ratio of the value of the exposure map at the aimpoint (expmap_aimpoint) to the value of the exposure map at the source position (expmap_source). The correction is done separately for each band using the exposure maps computed at energies of $1.0 \mathrm{keV}$ (soft band) and of $4.0 \mathrm{keV}$ (hard band). In this way the source count rate corresponds to the count rate that the source would have if it were observed at the aimpoint. The soft and hard conversion factors $(K)$ from counts $\left(\operatorname{cts~s}^{-1}\right)$ to X-ray fluxes (erg cm${ }^{-2} \mathrm{~s}^{-1}$ ) were derived at the aimpoint using the response matrices of the detector at this position. Such conversion factors were computed assuming an absorbed power-law spectrum with a photon index $\Gamma=1.7$ (Mushotzky 1984; Wilkes \& Elvis 1987) and assuming for the hydrogen column density, $N_{\mathrm{H}}$, the Galactic value along the line of sight at the source position (see Table 1, Col. 9, and Table 3, Col. 4).

The soft and hard X-ray source fluxes were calculated as

$S=\frac{C}{\tau} \times \frac{\text { expmap_aimpoint }}{\text { expmap_source }} \times K=\frac{C}{\tau} \times V \times K$

where $C$ are the net counts, $\tau$ the flare-corrected exposure time, $V$ the vignetting correction factor, and $K$ is the conversion factor from counts $\mathrm{s}^{-1}$ to $\mathrm{X}$-ray fluxes in $\mathrm{erg} \mathrm{cm}^{-2} \mathrm{~s}^{-1}$ appropriate for each energy band. The conversion factors allowed us to convert the $0.5-2.0 \mathrm{keV}$ band count rate to the observed X-ray fluxes in the same band and the $2.0-7.0 \mathrm{keV}$ band count rate to the observed X-ray flux in the $2.0-10.0 \mathrm{keV}$ band.

Flux uncertainties were estimated taking into account the error on the net counts $\left(\sigma_{\mathrm{NC}}\right)$ computed as the square root of the total observed counts in the "source cell", i.e. $\sqrt{C+B}$, and the error on the conversion factor, $\sigma_{K}$, due to the assumed different power-law models. The uncertainties on the conversion factor reflect the range of possible values for the effective photon index: $\Gamma=1.4-2.0$. The error on the flux then becomes:

$$
\begin{aligned}
\sigma_{S} & =\sqrt{\sigma_{\mathrm{NC}}^{2} \times\left(\frac{V \times K}{\tau}\right)^{2}+\sigma_{K}^{2} \times\left(\frac{C \times V}{\tau}\right)^{2}} \\
& =\sqrt{S^{2} \times\left(\frac{\sigma_{\mathrm{NC}}^{2}}{C^{2}}+\frac{\sigma_{K}^{2}}{K^{2}}\right)}
\end{aligned}
$$

The soft observed (i.e. absorbed) X-ray flux used to derived the source counts were multiplied by the factor, $\mathrm{c}_{N_{\mathrm{H}}}$ (Table 2, Col. 3) to obtain the Galactic unabsorbed X-ray flux. The correction factors were derived taking into account the Galactic hydrogen column density, $N_{\mathrm{H}}$, along the line of sight of each cluster (Table 1, Col. 9) and of each void of cluster field (Table 3, Col. 4).

\section{Appendix C: Sky coverage}

In Sect. 5 we derive the source counts (or $\log N-\log S$ ) for the point sources detected in the cluster regions. An important ingredient for the $\log N-\log S$ is the determination of the sky coverage. The "sky coverage" is the area of sky sensitive down to a given flux limit as a function of the flux density. To estimate 
the sky coverage, $\Omega$, we constructed a flux limit map, indicating the flux of the faintest source that would have been included in our source list at each position of the ACIS chip within the region of the cluster. The flux limit map has been constructed to account for the following effects:

- instrumental effects, such as vignetting, or increase of the point spread function size with off-axis angle. The sensitivity of the ACIS detectors varies significantly across the field of view;

- background effects. The background considered also includes the extended emission from the cluster's hot gas, since we detected sources within the region occupied by the clusters.

Following Eq. (B.1) the sensitivity limit $\left(S_{\lim }\right.$ in $\left.\operatorname{erg~} \mathrm{cm}^{-2} \mathrm{~s}^{-1}\right)$ at each detector position was defined as:

$S_{\lim }=\frac{C_{\lim }}{\tau} \times V \times K$

where $C_{\lim }$ are the net counts derived as in Eq. (C.2).

For the chosen signal-to-noise ratio $(S / N=3$; Sect. 4) one obtains from Eq. (1)

$C_{\lim }=3 \times\left(1+\sqrt{0.75+B_{\mathrm{avg}} \times A}\right)$

where $B_{\text {avg }}$ is the average background counts per pixel, $A$ the area expressed as the number of pixels covered by the source cell, and $B_{\text {avg }} \times A$ is the local background counts within the source region. The estimate of this area $A$ takes into account the degradation of the Chandra PSF, which increases with the off-axis angle. To estimate the local background (inclusive of the cluster extended emission) and the vignetting factor at each position of the chip, we built both a background and an exposure map of the same size and resolution for both the soft and the hard energy bands. The map of the effective background plus the extended cluster emission were obtained as follows:

1. All identified point sources were subtracted from the soft and hard images. The resulting "holes" were filled with pixel values sampled from the Poisson distribution whose mean and standard deviation equalled that of the surrounding background pixels (using the CIAO tool dmfilth);

2. These source-free images were binned by $32 \times 32$ pixels, so that each new pixel covers a linear size of $\sim 15.7^{\prime \prime}$. This is a good compromise between the resolution needed to smooth the very local background variations and at the same time to sample the variations due to the different emission from the cluster;

The exposure maps of the same size and binned by $32 \times 32$ pixels were constructed at energies of $1.0 \mathrm{keV}$ (soft) and $4.0 \mathrm{keV}$ (hard). The values corresponding to each image bin $(32 \times 32$ original pixels) within the region of the cluster allowed the calculation of the local average background per original pixel and the median exposure at the position of the bin. The last step in calculating $S_{\text {lim }}$ is an estimate of $A$. To do this it is necessary to study the increasing apparent size of the detected sources as a function of the off-axis angle. The size of a detected point source equals, by definition, the size of the Chandra PSF, which depends largely on the source's angular distance from the optical axis and, to some extent, on source energy. We studied and interpolated the increasing apparent size of the detected sources as a function of the off-axis angle separately in the soft and hard energy bands.

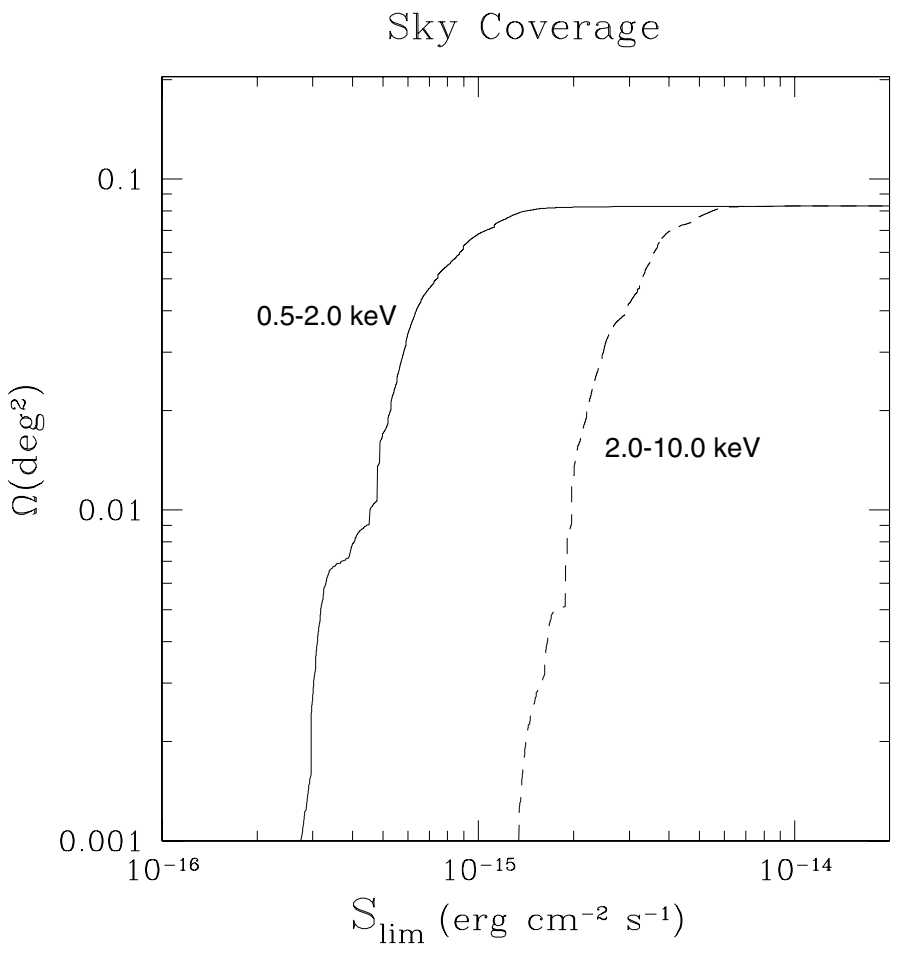

Fig. C.1. Sky coverage (area covered vs. flux limit) for the soft (solid line) and hard (dashed line) energy bands computed for the area covered by the 18 clusters of the sample.

For all the clusters we estimated the flux limit corresponding to each region. The sky area available at a given flux limit is then simply the sum of all the regions whose minimum detectable flux is lower than $S_{\text {lim. }}$. The total area covered by the "cluster survey" is $\sim 0.083 \mathrm{deg}^{2}$. In order to prevent incompleteness effects at the faint end of the source counts, we considered only those sources for each cluster with a flux larger than the flux corresponding to $20 \%$ of the total sky area covered by each cluster. Figure C. 1 shows the sky coverage for the eighteen clusters of our sample computed for the two energy bands. The steplike features visible in the sky coverage are given by the $20 \%$ cut described above. Note that the soft fluxes used to derived the soft-band sky coverage are unabsorbed fluxes, corrected for the the Galactic hydrogen column density along the line of sight of each field. 